\title{
Combinatorial synthesis of peptoid arrays via laser-based stacking of multiple polymer nanolayers
}

Daniela. S. Mattes ${ }^{[a, b]} *$, Bettina Streit ${ }^{[a, c]}$, Dhaka R. Bhandari ${ }^{[d]}$, Juliane Greifenstein ${ }^{[a]}$, Tobias C. Foertsch ${ }^{[a]}$, Stephan W. Münch ${ }^{[b]}$, Barbara Ridder ${ }^{[b]}$, Clemens v. Bojničić-Kninski ${ }^{[a]}$, Alexander Nesterov-Mueller ${ }^{[a]}$, Bernhard Spengler $^{[d]}$, Ute Schepers ${ }^{[c]}$, Stefan Bräse ${ }^{[b, c]}$, Felix F Loeffler ${ }^{[e] *}$ and Frank Breitling $^{[a] *}$

[a] D. S. Mattes, B. Steit, Dr. C. v. Bojničić-Kninski, Dr. B. Ridder, Prof. A. Nesterov-Mueller, Prof. F. Breitling

Institute of Microstructure Technology (IMT), Karlsruhe Institute of Technology (KIT), Hermann-vonHelmholtz-Platz 1, 76344 Eggenstein-Leopoldshafen, Germany

E-mail: daniela.mattes@kit.edu, frank.breitling@kit.edu

[b] D. S. Mattes, S. W. Münch, Prof. S. Bräse

Institute of Organic Chemistry, Karlsruhe Institute of Technology (KIT), Fritz-Haber-Weg 6, 76131

Karlsruhe, Germany

[c] Prof. S. Bräse, Prof. U. Schepers, B. Streit

Institute of Toxicology and Genetics, Karlsruhe, Institute of Technology (KIT), Hermann-von

Helmholtz-Platz 1, 76344 Eggenstein-Leopoldshafen, Germany

[d] Dr. D. R. Bhandari, Prof. B. Spengler

Institute of Inorganic and Analytical chemistry, Justus-Liebig University Giessen, Heinrich-Buff-Ring 17, 35392 Giessen, Germany

[e] Dr. F. F. Loeffler

Max Planck Institute of Colloids and Interfaces, Am Mühlenberg 1, 14476 Potsdam, Germany

E-mail: felix.loeffler@mpikg.mpg.de

Keywords: Molecule arrays, high-throughput screening, submonomer method, MALDI MS imaging, laser-induced forward transfer 
Here, we report the combinatorial synthesis of molecule arrays via a laser-assisted process. Lasertransferred polymer nanolayers with embedded monomers, activators, or bases can be reliably stacked on top of each other, spot-by-spot, to synthesize molecule arrays. These various chemicals in the nanometer thin layers are mixed by heat or solvent vapor, inducing coupling reactions. As an example, we generated peptoid arrays with a density of 10000 spots $/ \mathrm{cm}^{2}$ with the sub-monomer or monomer method. Moreover, we verified successful reactions spot-by-spot, by laser-transferring MALDI-matrix (Matrix-assisted laser desorption/ionization), followed by MALDI mass spectrometry imaging.

High-density molecule arrays allow for rapid and parallelized screenings. The well-automated - yet relatively large scaled - synthesis of oligonucleotides ${ }^{[1]}$, peptides/proteins ${ }^{[2,3]}$, and carbohydrates ${ }^{[4]}$, enabled the synthesis and subsequent arrangement of these molecules as small spots on a microarray surface. The advent of in situ synthesis of high-density molecule arrays ${ }^{[1,3,5]}$ promises the highly parallelized synthesis of minute amounts of molecules directly in the microarray format. However, these techniques require a high spatial precision, robust chemical strategies, high yields, and they should offer quality control. ${ }^{[6]}$ Therefore, new methods are required, which offer various chemical synthesis strategies for high-density arrays of different types of molecules, for example, to find new catalysts ${ }^{[7]}$ or polymers. $^{[8,9]}$

For peptide arrays, which are used to investigate protein-peptide interactions, several methods have been developed. ${ }^{[2]}$

Recently, we developed a laser-based synthesis method for peptide array synthesis (Supporting Information Figure S1A). ${ }^{[10]}$ In this system, various donor slides, coated with a polymer matrix, containing a pre-activated amino acid, are consecutively placed on top of an acceptor slide, where the coupling takes place. Short laser pulses transfer nanometer thin solid polymer spots to selected areas of the acceptor surface, thereby, structuring the acceptor slide with the reagents. Heat or solvent vapor induces melting and mixing of the transferred solid materials, thereby, activating the embedded reagents, before diffusing to the surface of the acceptor slide, where they react with negligible lateral diffusion to "their" spot. ${ }^{[10]}$ 
Some groups have adapted peptide array methods, such as the SPOT-synthesis ${ }^{[9,}$ 11-13] or photolithographic techniques ${ }^{[14]}$ for peptoid array synthesis. Peptoids can mimic antibody-binding peptides, ${ }^{[15]}$ and pharmaceutical companies are especially interested in peptidomimetics ${ }^{[16]}$ as preferred protein-binding agents. ${ }^{[17]}$ Compared to peptides, peptoids are resistant to proteolytic cleavage, ${ }^{[18]}$ they are more soluble, and they penetrate membranes more easily. ${ }^{[19]}$ They can be used in diagnostics, therapeutics, e.g. antimicrobial peptoids, ${ }^{[20]}$ or even as transcription factor mimetics. ${ }^{[21]}$

The first combinatorial approach to peptoid synthesis was reported in 1992 by Bartlett et al. ${ }^{[22]}$, following the submonomer method of Zuckermann ${ }^{[23]}$ on a solid support. Peptoids were synthesized on a resin by coupling bromoacetic acid $(\mathrm{BrAcOH})$ with $N, N^{\prime}$-diisopropylcarbodiimide (DIC) ${ }^{[22]}$ and displacing the bromide with an amine in a nucleophilic substitution (Supporting Information Figure S1B). A variant SPOT-synthesis was used to first spot bromoacetic acid and an activation reagent for the acylation, and then the amines to displace the bromide. By repeating this process, an array of peptoids was synthesized. One drawback of SPOT synthesis is the low density of resulting peptoid arrays $\left(\sim 25\right.$ spots per $\left.\mathrm{cm}^{2}\right)$. Another peptoid synthesis approach uses Fmoc-protected $N$-substituted glycines (i.e. monomer building blocks of the peptoid). ${ }^{[24]}$ Analogous to peptide array synthesis, an activation reagent is necessary e.g. PyBOP for coupling of the monomers (Supporting Information Figure S1C). Compared to the sub-monomer method, this method gives high yields, fewer side products, easily stainable $\mathrm{N}$-termini, and enables reaction control via UV-Vis spectroscopy during Fmocdeprotection (Fulvene-piperidine adduct, $301 \mathrm{~nm}$ ). A drawback, however, is the lack of commercially available amines when compared to the submonomer method. ${ }^{[24]}$

Higher densities were achieved by spotting pre-synthetized peptoids, which is traded, however, for prohibitive costs. ${ }^{[25]}$ Lithographic synthesis was used to synthesize peptoid arrays, with theoretically up to 100000 different peptoids (no exact spot density mentioned). ${ }^{[14]}$ However, due to the inherent low cleavage yield of photo-protecting groups and the serial coupling of monomers, it is difficult to generate high-quality peptoid arrays. Furthermore, this makes it difficult to use the large variety of existing amines (> 10000 amines $^{[15]}$ ). For spot-by-spot validation of a successful in situ peptoid synthesis in the 
array format, spatially resolved MALDI mass spectrometry imaging (MSI) ${ }^{[26]}$ certainly is the method of choice. In order to desorb and ionize the synthesized molecules the spots must be covered with a thin layer of MALDI matrix material. This has been done by various ablation e.g. spray, ${ }^{[27]}$ sublimation, ${ }^{[28]}$ spotting $^{[29]}$ or printing ${ }^{[21]}$ are used to overlay molecules with a MALDI matrix.

To demonstrate the applicability and compatibility of this laser-based polymer-transfer method for other chemical reactions, we generated peptoid arrays, which require different chemicals to be lasertransferred in various layers. Afterwards, we laser-transferred the MALDI-matrix spot-by-spot, to validate the synthesis via MALDI mass spectrometry imaging (MALDI-MSI).

\section{Results and Discussion}

\section{Sub-monomer method}

We used the laser system to stack spot-by-spot nanometer-thin layers of bromoacetic acid on top of the activation reagent DIC (both embedded in a polymer matrix material), where they could couple to the free amines of a $\beta$-Alanine functionalized 10:90 PEGMA (poly(ethylenglycol)methacrylate)-co-MMA (methyl(metacrylate) $)^{[30]}$ acceptor surface (Figure 1). Next, a heating step at $90{ }^{\circ} \mathrm{C}$ for $1 \mathrm{~h}$, induced diffusion, mixing, and coupling. For the final step of the first cycle - the nucleophilic substitution - we used another donor slide that featured the embedded amine. Again, short laser pulses transferred tiny material spots at selected areas to the acceptor in order to substitute the bromide with the amine. The desired chain length was reached through repetition of these steps. Thereby, we adapted the submonomer method of Zuckermann to our laser-based approach to synthesize 1600 peptoid spots.

For initial MALDI-MS validation, peptoids were synthesized on a Rink-Amide-Linker functionalized surface, which was reacted with a glycine, offering a free amine. First, we transferred DIC to the surface and then bromoacetic acid. For a more reliable coupling reaction, we added another layer of DIC on top. The donor slides, bearing a laser absorption layer (polyimide foil), were prepared by spin coating a mixture of each substance with a polymer dissolved in dichloromethane (DCM). A good solvation of 
the reagents and the polymer in the solvent (DCM) is important for a homogenous coupling reaction. After a heating $\left(80-90^{\circ} \mathrm{C}\right)$ and washing step, we transferred the different amines for the bromide substitution. Repeating the heating and washing steps once again, resulted in an $N$-substituted glycine. For amines with boiling points below $90{ }^{\circ} \mathrm{C}$, coupling can be performed in solvent vapor under a petri dish (instead of heating), dissolving the polymer matrix, and allowing the molecule to couple to the surface. The crude product was cleaved from the surface with trifluoroacetic acid (TFA), triisobutylsilane (TIBS), and $\mathrm{H}_{2} \mathrm{O}$ in dichloromethane (DCM) for MALDI-MS analysis (Supporting Information 2.1.1).

In addition, we also analyzed different modes of bromoacetic acid activation using our approach. ${ }^{[2]}$ The most successful was bromoacetic-2,4-dinitrophenylester (-ODNP) (Supporting Information 3.2.1). By adjusting the laser parameters, we could control the yield and spot size, ${ }^{[10]}$ since the irradiation period directly correlates with the amount of transferred material (Supporting Information 3.2.1).

Amine volatility, nucleophilicity, and steric requirements have been already described for the SPOT synthesis. ${ }^{[2]}$ However, we had to improve the substitution reaction to achieve high coupling yields (Supporting Information 3.2.2). Peptoid arrays with various amines were synthesized and detected via fluorescence or MALDI-MS. A peptoid array $\left(400 \mathrm{spots} / \mathrm{cm}^{2}\right)$ was generated in an A (red) B (green) chessboard design (Figure 2A), and a pitch of $250 \mu \mathrm{m}$ was reached in another array (Figure 2B). Moreover, we synthesized peptoids and validated them via MALDI-MSI ${ }^{[31]}$ in a spatially resolved manner (Figure 2 C, D, E, F; Supporting Information 2.3).

We can use a Photo-Linker functionalized surface, and cleave the peptoids via UV-irradiation, without losing spatial resolution. ${ }^{[25]}$ Alternatively, a Rink-Linker ${ }^{[26]}$ was considered, however, spots diffused during cleavage with TFA vapor. Hence, we employed the laser-based method to directly transfer the MALDI-matrix to the spots (Figure 3F). To the best of our knowledge, this is the first report of a laserbased method to coat a spot with a MALDI-matrix. The thickness of the MALDI-matrix can be adjusted in the nm-range via the duration of irradiation. Due to the lack of a solvent, no lateral diffusion occurs. 


\section{Monomer method}

Next, we also followed the monomer method to synthesize 10000 peptoid spots per $\mathrm{cm}^{2}$ (Figure 3A). Advantages of the monomer method for the laser-based approach are the stability of the compounds in the donor slides (up to 5 months), the possibility to reuse them up to 20 times, ${ }^{[23]}$ as well as the use of amines with boiling points close to room temperature. However, the disadvantage is the labor-intensive synthesis of the Fmoc- $N$-protected glycines, which restricts the diversity of possible peptoids.

Again, we stacked multiple nanometer-thin spots on top of each other in a pattern onto the acceptor slide. Initially, we transferred Fmoc- $N$-substituted glycine from the first donor slide, then, DIC from a second donor, and HOBt from a third donor. After the heating step, to induce the coupling, unreacted groups were capped. Finally, the Fmoc-groups are removed, to yield free amines on the surface for the next peptoid bond synthesis. By repeating these steps, we generated arrays with peptoids of the desired length.

The final peptoids were detected via fluorescence (Figure 3B, C). For the validation via MALDI-MS, a 3-mer peptoid (Figure 3D) was synthesized. Moreover, for the activation of the Fmoc- $N$-substituted glycine, we used PyBOP, DIPEA, and HOBt. The chemicals were laser-transferred in different layers on top of each other: Fmoc- $N$-substituted glycine (first donor), PyBOP (second donor), DIPEA (third donor) and HOBt (fourth donor) (Figure $3 \mathrm{E}$ ). To demonstrate the diffusion of the Fmoc- $N$-substituted glycine through three deposited layers, we laser-transferred the monomer as the fourth layer, which is the furthest away from the surface (Figure $3 \mathrm{~F}$ ). This order of layer deposition also gave a positive coupling result.

In conclusion, we synthesized peptoids with our solid-material-based approach in the array format, with spot densities of 1600 peptoids $/ \mathrm{cm}^{2}$ (submonomer method) and 10000 peptoids $/ \mathrm{cm}^{2}$ (monomer method). Moreover, we used the same method to cover acceptor surfaces with a nanolayer spot of MALDI-matrix, which allowed us to determine the molecular weight of synthesized molecules in individual spots by MALDI-MSI. 
This method shows great potential in combinatorial chemistry, because of its high flexibility and versatility. In principle, any microarray could be validated with our laser-transferred matrix transfer approach and MALDI-MSI. This high-density approach offers unprecedented capabilities to screen peptoids for binding activities and can be used for the discovery of potential therapeutics, such as biomarkers or antimicrobials. The flexibility of our method should enable other array-based chemistry, due to the stacking of nanometer-thin layers of various chemicals, embedded in a polymer and could usher in a new field for high-throughput chemical synthesis, validation, and screening.

Image editing: The contrast of the pictures was enhanced with Image J 1.46v or Irfan view 4.35.

\section{Supporting Information}

Supporting Information is available from the Wiley Online Library or from the author.

Acknowledgements: We thank M. Schnölzer and S. Fiedler from the German Cancer Research Center for MALDI-ToF measurements, B. Kühl from the Institute of Functional Interfaces (KIT) for supporting the MALDI-ToF measurements, S. Vanderheiden-Schroen from the Institute of Toxicology and Genetics (KIT) for support in ESI-MS, A. Schneider from the Institute of Organic chemistry (KIT) for the synthesis of the amines and A. Taruschkina from the Institute of Microstructure Technology (KIT) for support in synthesis. For financial support, the authors acknowledge the ERC [ERC PoC no. 766695], the BMBF [grant nos. 03EK3030A, 031A095C, 13XP5050A], GRK 2039, funded by the DFG, and the Max Planck Society. The authors declare competing financial interests: F. Breitling is shareholder of PEPperPRINT GmbH. A. Nesterov-Mueller, F. Breitling, and F.F. Loeffler are named on pending patent applications related to molecule array synthesis PCT/EP2014/001046 and US 20160082406.

This is the pre-peer reviewed version of the following article: [Mattes, D. S.; Streit, B.; Bhandari, D. R.; Greifenstein, J.; Foertsch, T. C.; Münch, S. W.; Ridder, B.; v. Bojničić-Kninski, C.; Nesterov-Mueller, A.; Spengler, B.; Schepers, U.; Bräse, S.; Loeffler, F. F.; Breitling, F. 2018. Macromolecular rapid communications, 40 (6), Art.Nr.: 1800533.], which has been published in final form at 
[doi:10.1002/marc.201800533 ]. This article may be used for non-commercial purposes in accordance

with Wiley Terms and Conditions for Use of Self-Archived Versions.

[1] R. J. Lipshutz, S. P. A. Fodor, T. R. Gingeras, D. J. Lockhart, Nature Genetics 1999, 21, 20.

[2] C. Katz, L. Levy-Beladev, S. Rotem-Bamberger, T. Rito, S. G. D. Rudiger, A. Friedler,

Chemical Society Reviews 2011, 40, 2131.

[3] L. C. Szymczak, H.-Y. Kuo, M. Mrksich, Analytical Chemistry 2018, 90, 266.

[4] T. Horlacher, P. H. Seeberger, Chemical Society Reviews 2008, 37, 1414; J. Voskuhl, J.

Brinkmann, P. Jonkheijm, Current Opinion in Chemical Biology 2014, 18, 1.

[5] A. Geissner, P. H. Seeberger, Annual Review of Analytical Chemistry 2016, 9, 223.

[6] G. Schneider, Nature Reviews Drug Discovery 2017, 17, 97.

[7] C. Peijun, D. R. D., F. Qun, G. D. M., G. Shenheng, M. E. W., P. D. M., S. Kyle, T. H. W., W.

W. Henry, Angewandte Chemie International Edition 1999, 38, 483.

[8] M. M. A. R., H. Richard, S. U. S., Macromolecular Rapid Communications 2004, 25, 21.

[9] A. W. Bosman, A. Heumann, G. Klaerner, D. Benoit, J. M. J. Fréchet, C. J. Hawker, Journal of the American Chemical Society 2001, 123, 6461.

[10] F. F. Loeffler, T. C. Foertsch, R. Popov, D. S. Mattes, M. Schlageter, M. Sedlmayr, B. Ridder, F. X. Dang, C. von Bojnicic-Kninski, L. K. Weber, A. Fischer, J. Greifenstein, V. Bykovskaya, I. Buliev, F. R. Bischoff, L. Hahn, M. A. Meier, S. Brase, A. K. Powell, T. S. Balaban, F. Breitling, A. NesterovMueller, Nat Commun 2016, 7, 11844.

[11] N. Heine, T. Ast, J. Schneider-Mergener, U. Reineke, L. Germeroth, H. Wenschuh, Tetrahedron 2003, 59, 9919.

[12] H. N. Heine, Humboldt-Universität Berlin 2000.

[13] A. C. Schneider, D. Fritz, J. K. Vasquez, S. B. L. Vollrath, H. E. Blackwell, S. Bräse, ACS Combinatorial Science 2017, 19, 715.

[14] S. Li, D. Bowerman, N. Marthandan, S. Klyza, K. J. Luebke, H. R. Garner, T. Kodadek, J Am Chem Soc 2004, 126, 4088.

[15] G. K. Olivier, A. Cho, B. Sanii, M. D. Connolly, H. Tran, R. N. Zuckermann, ACS Nano 2013, $7,9276$.

[16] A. Rabocchi, A.Guarna, Peptidomimetics in organic and medicinal chemistry: the art of transforming peptides in drugs., Vol. 89, John Wiley \& Sons Ltd,, Chichester 2014.

[17] P. G. Alluri, M. M. Reddy, K. Bachhawat-Sikder, H. J. Olivos, T. Kodadek, J Am Chem Soc 2003, 125, 13995.

[18] S. M. Miller, R. J. Simon, S. Ng, R. N. Zuckermann, J. M. Kerr, W. H. Moos, Bioorganic \& Medicinal Chemistry Letters 1994, 4, 2657; R. J. S. Susan M. Miller, Simon Ng, Ronald N.

Zuckermann, Janice M. Kerr, Walter H. Molina, Drug Dev. Res. 1995, 35, 20.

[19] K. H. A. Lau, Biomater Sci-Uk 2014, 2, 627.

[20] A. R. Statz, J. P. Park, N. P. Chongsiriwatana, A. E. Barron, P. B. Messersmith, Biofouling 2008, 24, 439; P. Mora, I. Masip, N. Cortés, R. Marquina, R. Merino, J. Merino, T. Carbonell, I. Mingarro, A. Messeguer, E. Pérez-Payá, Journal of Medicinal Chemistry 2005, 48, 1265.

[21] X. Xiao, P. Yu, H.-S. Lim, D. Sikder, T. Kodadek, Angewandte Chemie International Edition 2007, 46, 2865.

[22] R. J. Simon, R. S. Kania, R. N. Zuckermann, V. D. Huebner, D. A. Jewell, S. Banville, S. Ng, L. Wang, S. Rosenberg, C. K. Marlowe, et al., Proceedings of the National Academy of Sciences of the United States of America 1992, 89, 9367.

[23] R. N. Zuckermann, J. M. Kerr, S. B. H. Kent, W. H. Moos, Journal of the American Chemical Society 1992, 114, 10646.

[24] J. A. W. Kruijtzer, L. J. F. Hofmeyer, W. Heerma, C. Versluis, R. M. J. Liskamp, Chem-Eur J $1998,4,1570$.

[25] http://www.raybiotech.com/files/manual/Peptoids/PA7-2800-2.pdf, 26.04.2017. 
[26] P. K. M. P. Greving, Z. Zhao, N. W. Woodbury Langmuir 2010, 26, 1456.

[27] H. Wei, K. Nolkrantz, D. H. Powell, J. H. Woods, M.-C. Ko, R. T. Kennedy, Rapid Communications in Mass Spectrometry 2004, 18, 1193.

[28] J. A. Hankin, R. M. Barkley, R. C. Murphy, Journal of the American Society for Mass Spectrometry 2007, 18, 1646.

[29] H.-R. Aerni, D. S. Cornett, R. M. Caprioli, Analytical Chemistry 2006, 78, 827.

[30] V. Stadler, R. Kirmse, M. Beyer, F. Breitling, T. Ludwig, F. R. Bischoff, Langmuir 2008, 24, 8151.

[31] D. R. Bhandari, T. Shen, A. Rompp, H. Zorn, B. Spengler, Analytical and bioanalytical chemistry 2014, 406, 695.

[32] J. Striffler, D. S. Mattes, S. Schillo, B. Munster, A. Palermo, B. Ridder, A. Welle, V. Trouillet, V. Stadler, G. Markovic, G. Proll, S. Brase, F. F. Loeffler, A. Nesterov-Muller, F. Breitling, Chemnanomat 2016, 2, 897.

[33] C. D. Hein, X.-M. Liu, D. Wang, Pharmaceutical research 2008, 25, 2216.

[34] H. Ammann, G. Dupuis, Can. J. Chem. 1988, 68, 1651; P. Cuatrecasas, M. Wilchek, C. B. Anfinsen, Proc. Natl. Acad. Sci. U. S. A. 1968, 61, 636. 


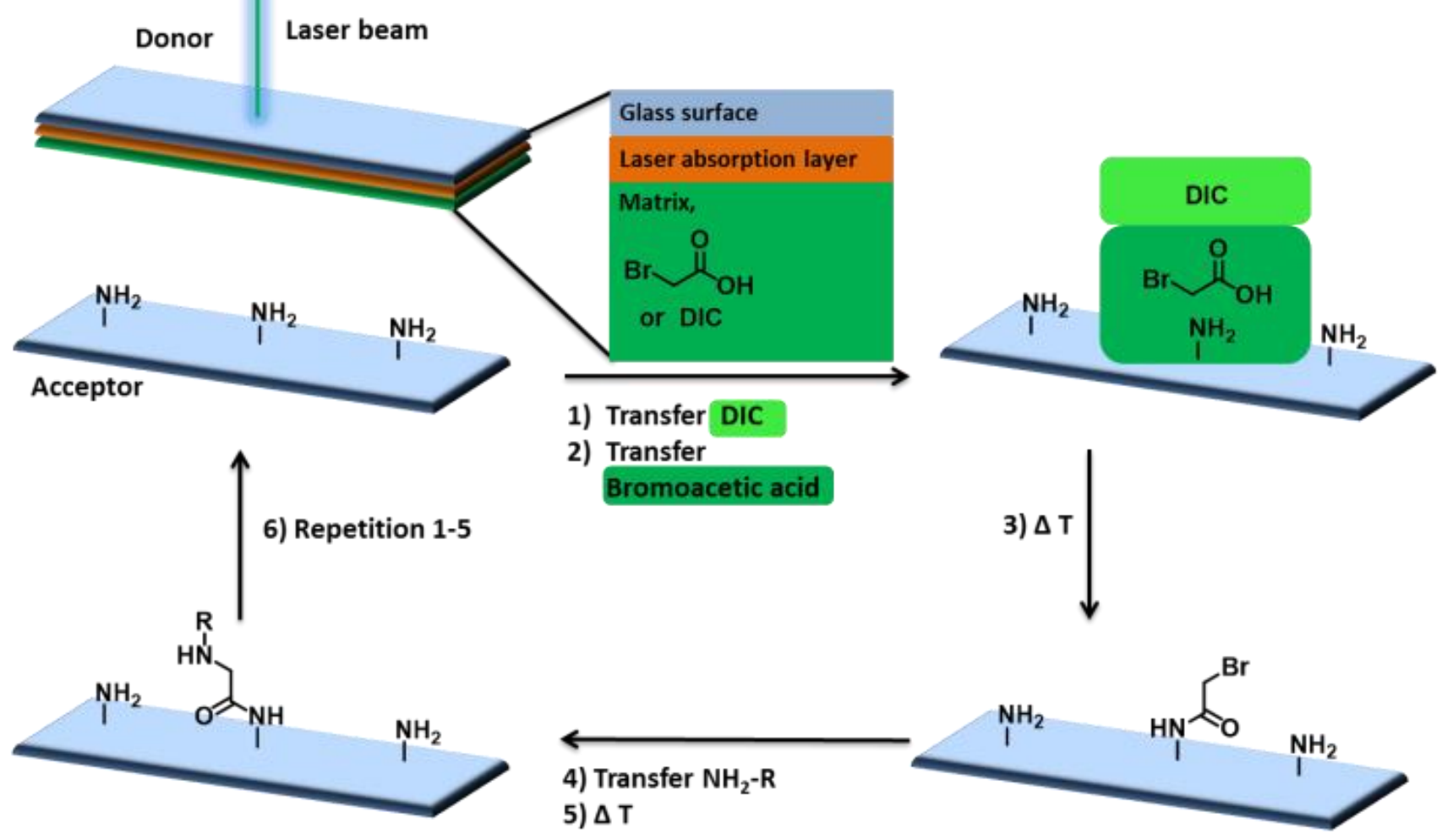

Figure 1. Synthesis of peptoid arrays with the submonomer method via laser-transferred nanometer-thin layers: 1, 2) DIC and bromoacetic acid are transferred to the surface (alternatively an active ester of bromoacetic acid). 3) Coupling in the oven. 4) Amine transfer. 5) Coupling in the oven or via solvent vapor. 6) Repetition 1-5. 


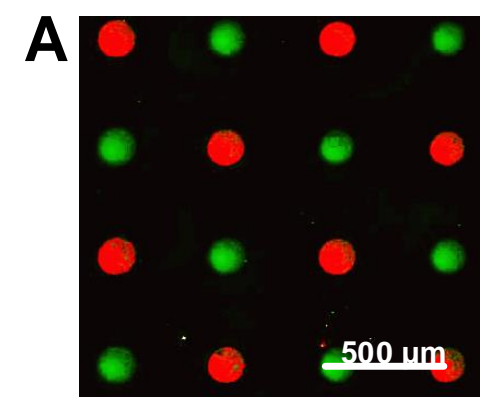

D<smiles>NC(=O)NCCNC(=O)CNC(=O)CNC(=O)CNC(=O)CNC(N)=O</smiles>

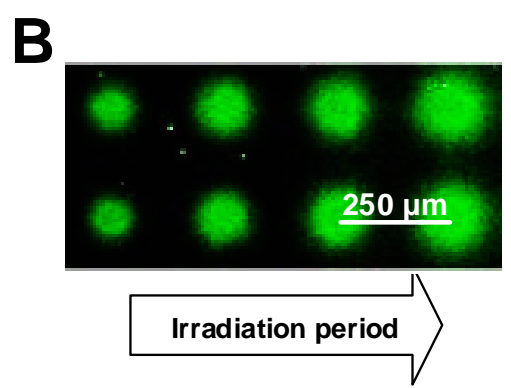

$\mathbf{E}$
C

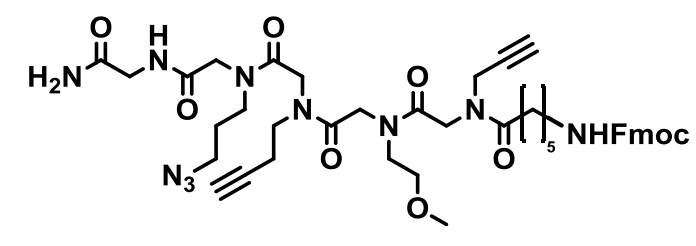

$\mathbf{F}$

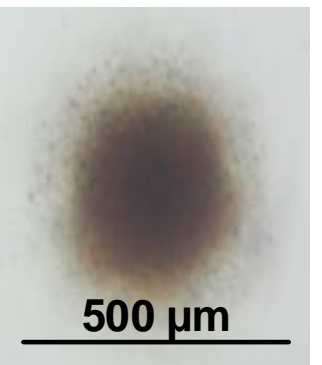

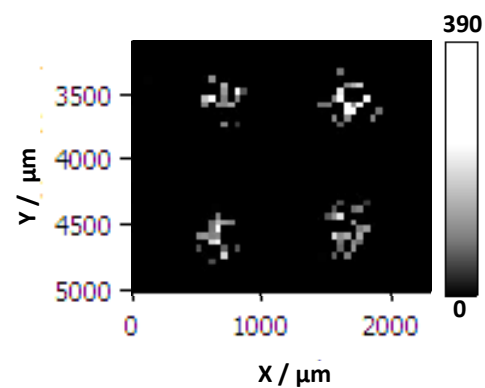

Figure 2. (A) Fluorescence image of a peptoid array in a chessboard pattern (pitch $500 \mu \mathrm{m}$ ); spot (red): Mono-boc-diaminobutane, labeled with DyLight650-NHS; Spot (green): tert-butyl-aminobutanoic acid, labeled with TAMRA-NHS. (B) Fluorescence image of a peptoid array, pitch $250 \mu \mathrm{m}$, pulse duration < $2.0 \mathrm{~ms}$; spots: bromoacetic acid-ODNP, aminobutyne; staining: TAMRA-azide. (C) Synthesized 4mer peptoid on a Rink-Amide-Linker following the standard protocol with BrAc-ODNP and amine coupling: azidopropylamine, aminobutyne, methoxyethylamine, and propargylamine. (D) Backbone of a peptoid, illustrating two different peptoids (different residues) on a Photo-Linker, functionalized with Fmoc-Gly-OPfp. Peptoid 1: TBDMS-aminoethanol (R1), aminobutyne (R2), methoxyethylamine (R3), propargylamine (R4) and Peptoid 2: TBDMS-aminoethanol (R1), methoxyethylamine (R2), aminobutyne (R3), propargylamine (R4) (E) Laser-transferred DHB/CHCA MALDI matrix. (F) MALDI-MSI $[\mathrm{M}+\mathrm{K}]^{+}$of the peptoids (see D). Lateral resolution was set to $50 \mu \mathrm{m} / \mathrm{pixel}$. The image was generated for the measured mass-to-charge-number $\mathrm{m} / \mathrm{z}=533.21016 \mathrm{u}+/-5 \mathrm{ppm}$. 

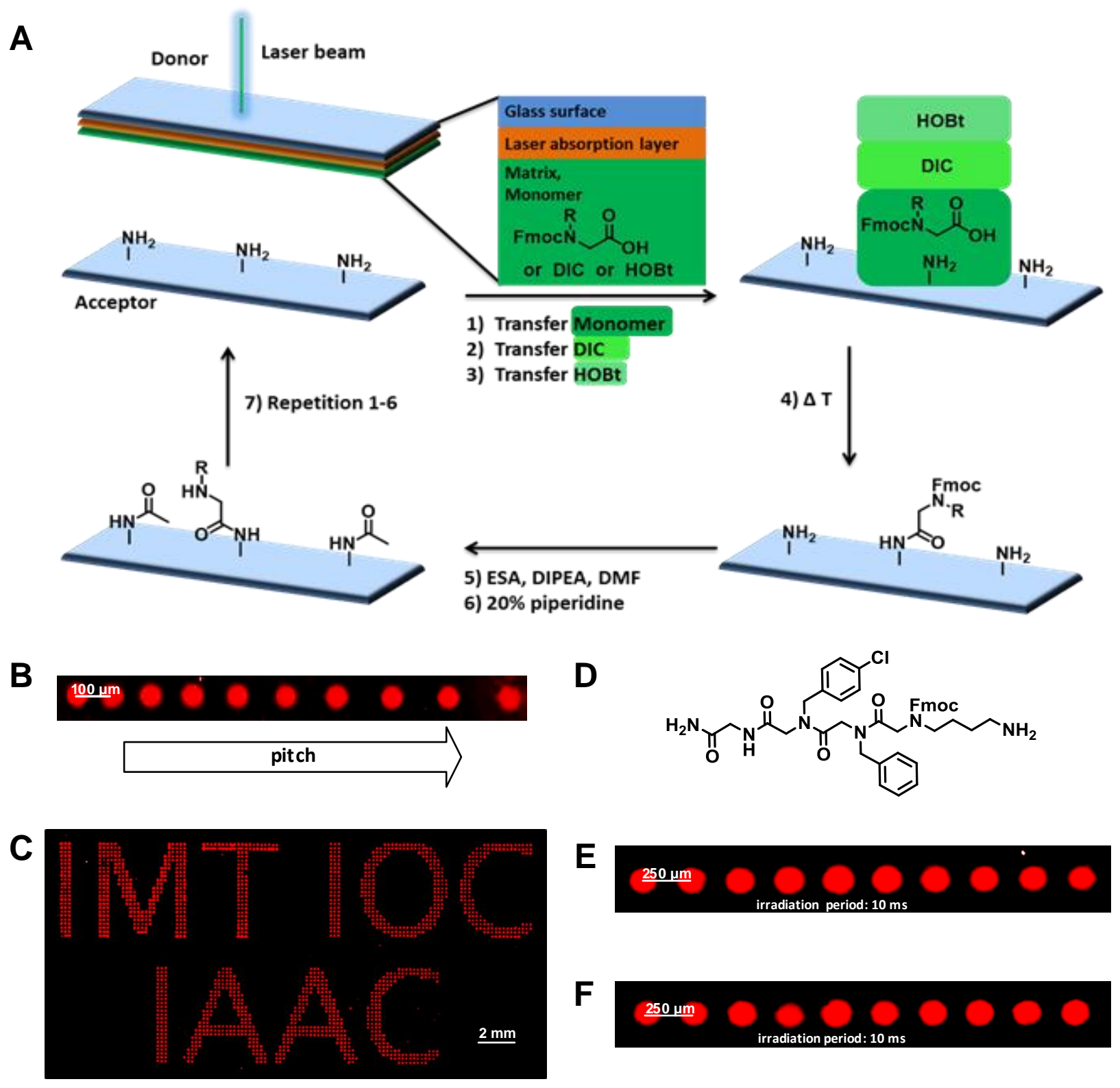

Figure 3. (A) Synthesis of peptoid arrays using the monomer method with laser-transferred nanometerthin layers: 1) Fmoc-N-protected glycine transfer. 2) DIC transfer. 3) HOBt transfer. 4) Coupling in the oven. 5) Acetylation. 6) Fmoc deprotection. 7) Repetition of steps 1-6. (B) Demonstration of spot resolution/separation $(100-172 \mu \mathrm{m}$, irradiation period $4 \mathrm{~ms})$. Fmoc- $N$ PheCl-OH coupling with DIC and HOBt, acetylation, Fmoc-deprotection, and biotin-OPfp coupling in DMF. Staining: streptavidinAtto647 (Supporting Information 2.4). (C) Coupling of 3 different monomers. IMT: Fmoc-Abg(Boc)OH, IOC: Fmoc- $N$ Phe-OH, and IAAC: Fmoc- $N$ PheOMe-OH (pitch $200 \mu \mathrm{m}$, laser duration $10 \mathrm{~ms}$ ) (Implementation see A). (D) 3mer Peptoid, monomers: Fmoc- $N \mathrm{PheCl}-\mathrm{OH}$, Fmoc- $N \mathrm{Phe}-\mathrm{OH}$, and Fmoc$\mathrm{Abg}(\mathrm{Boc})-\mathrm{OH}$ (Supporting Information 2.1). (E) Coupling of Fmoc-NPhe-OH (first donor) with PyBOP (second donor), DIPEA (third donor), HOBt (fourth donor), acetylation, Fmoc-deprotection, and biotinOPfp coupling in DMF. Staining: streptavidin-Atto647. (F) Coupling of Fmoc- $N$ Phe-OH (fourth donor), with PyBOP (first donor), DIPEA (second donor), HOBt (third donor) acetylation, Fmoc-deprotection, and biotin-OPfp coupling in DMF. Staining: streptavidin-Atto647. 


\section{Supporting Information}

\section{Combinatorial synthesis of peptoid arrays via laser-based stacking of multiple polymer nanolayers}

Daniela. S. Mattes ${ }^{[a, b]} *$, Bettina Streit ${ }^{[a, c]}$, Dhaka R. Bhandari ${ }^{[d]}$, Juliane Greifenstein ${ }^{[a]}$, Tobias C.

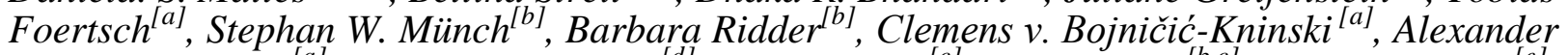
Nesterov-Mueller ${ }^{[a]}$, Bernhard Spengler ${ }^{[d]}$, Ute Schepers ${ }^{[c]}$, Stefan Bräse ${ }^{[b, c]}$, Felix F Loeffler ${ }^{[l]} *$ and Frank Breitling ${ }^{[a] *}$

Abstract: Here, we report the combinatorial synthesis of molecule arrays via a laser-assisted process. Laser-transferred polymer nanolayers with embedded monomers, activators, or bases can be reliably stacked on top of each other, spot-by-spot, to synthesize molecule arrays. These various chemicals in the nanometer thin layers are mixed by heat or solvent vapor, inducing coupling reactions. As an example, we generated peptoid arrays with a density of 10000 spots $/ \mathrm{cm}^{2}$ with the sub-monomer or monomer method. Moreover, we verified successful reactions spot-by-spot, by laser-transferring MALDI-matrix (Matrix-assisted laser desorption/ionization), followed by MALDI mass spectrometry imaging.

\section{Table of Contents}

\section{Experimental Procedure}

1.1 Fmoc-deprotection

1.2 Standard washing protocol and drying process (SWP)

1.3 Acetylation of the free amines

1.4 Side-chain deprotection

1.5 Surface functionalization via amidation

1.6 Functionalization of surfaces with the Fmoc-Rink-Amid-Linker

1.7 Cleavage of the crude product

1.8 Preparation of the donor slide for the laser-induced forward transfer (PDS) 
1.9 Combinatorial laser-induced forward transfer (cLIFT)

1.10 Sub-monomer method

$1.11 \quad$ Monomer method

1.12 Pre-structuring with Fmoc-Gly-OPfp

1.13 Synthesis of peptoids Figure 2A; Spot A and B

1.14 Synthesis of peptoids Figure 2B

1.15 Synthesis of peptoids Figure 2C

1.16 Synthesis of peptoids in Figure 2D

1.17 Synthesis of peptoids Figure 3B

1.18 Synthesis of Peptoid in Figure 3C, Logos of IMT, IOC, IAAC

1.19 Synthesis of peptoid Figure 3D

1.20 Synthesis of peptoid Figure 3E

1.21 Synthesis of peptoid in Figure 5F

1.22 Synthesis of peptoid A1

1.23 Synthesis of peptoid A2

1.24 Synthesis of peptoid A4

1.25 Fluorescent staining protocol

1.26 Synthesis of bromoacetic succinimide ester and bromoacetic pentafluorophenylester

1.27 Synthesis of bromoacetic 2,4-dinitrophenylester

1.28 Synthesis of Fmoc- $N$ Phe-OH

1.29 Synthesis of Fmoc- $N \mathrm{PheCl}-\mathrm{OH}$

\section{Synthesis of arrays}

\section{Results}

3.1 Mass spectrometry (MALDI) of peptoids (crude product) 
3.2 Optimization

3.3 Mass spectrometry (MALDI imaging) of peptoids

3.4 Pitch experiment with the monomer method

\section{Materials}

\section{Analytical Methods}

6 cLIFT setup

7 Fluorescence scanner

8 Author contributions 


\section{Experimental Procedure}

1.1 Fmoc-deprotection ${ }^{[32]}$ : Fmoc-protecting groups were deprotected by incubating the 10:90-poly(ethylene methacrylate (PEGMA)-co-methyl methacrylate (MMA) surface in a prepared solution of piperidine/(dimethylformamide) DMF (dry) (20\%:80\% (v/v)) for $20 \mathrm{~min}$ under shaking. Afterwards, the standard washing protocol (SWP) was performed (see 1.2).

1.2 Standard washing protocol and drying process (SWP) ${ }^{[32]}$ : The 10:90-PEGMA-coMMA surface was washed on a linear shaker in $3 \times 5 \min \mathrm{DMF}, 1 \times 2$ min methanol $(\mathrm{MeOH})$ and $1 \times 1$ min dichloromethane $(\mathrm{DCM})$, each time the solvent was refreshed. Finally, the slides were dried under a stream of argon.

1.3 Acetylation of the free amines ${ }^{[32]}$ : The 10:90-PEGMA-co-MMA surface was acetylated by rocking the slide with a solution of acetic anhydride (ESA)/diisopropylethylamine (DIPEA) / DMF (dry) (10\%:20\%:70\% (v/v)) under argon atmosphere for $4 \mathrm{~h}-16 \mathrm{~h}$, followed by the SWP.

1.4 Side-chain deprotection ${ }^{[32]}$ : The side chain protecting groups were removed by rocking the arrays in a solution of DCM/trifluoroacetic acid (TFA)/triisobutylsilane (TIBS)/ultrapure water (44\%:51\%:3\%:2\% (v/v/v/v)) for $90 \mathrm{~min}$, followed by the SWP.

1.5 Surface functionalization via amidation ${ }^{[32]}$ : A functionalized 10:90-PEGMA-co-MMA surface was placed in a petri dish in a desiccator and $1.00 \mathrm{~mL}$ of $50.0 \mathrm{mM}$ solution of carboxy acid, $N, N^{\prime}$-diisopropylcarbodiimide (DIC), Ethyl (hydroxyimino)cyanoacetate (Oxyma Pure) was pipetted on the slide overnight, followed by the SWP. Alternatively, a functionalized 10:90-PEGMA-co-MMA surface was placed in a petri dish in a desiccator and $1.00 \mathrm{~mL}$ of 
$50.0 \mathrm{mM}$ solution of Fmoc-Photo-Linker with 2-(1H-benzotriazol-1-yl)-1,1,3,3tetramethyluronium-hexafluorophosphat (HBTU), DIPEA, hydroxybenzotriazole (HOBt) in DMF was pipetted on the slide overnight, followed by the SWP. Alternatively, a functionalized 10:90-PEGMA-co-MMA surface was placed in a petri dish in a desiccator and $1.0 \mathrm{~mL}$ of $50.0 \mathrm{mM}$ solution of Fmoc-Gly-OPfp was pipetted on the slide overnight, followed by the SWP. Alternatively, a functionalized 10:90-PEGMA-co-MMA surface was placed in a petri dish in a desiccator and $1.00 \mathrm{~mL}$ of $50.0 \mathrm{mM}$ solution of Biotin-OPfp was pipetted on the slide overnight, followed by the SWP.

\subsection{Functionalization of the surfaces with the Fmoc-Rink-Amid-Linker ${ }^{[10]}$ : A} functionalized 10:90-PEGMA-co-MMA surface was placed in a petri dish in a desiccator. $250 \mu \mathrm{L}$ of a solution of $200 \mathrm{mM}$ Fmoc-Rink-Amide-Linker, $200 \mathrm{mM}$ pentafluorophenol and $200 \mathrm{mM}$ DIC in DMF (dry) was pipetted on top of the slide and a microscope slide was placed on top of the surface. The sandwich was left to react overnight under argon, followed by the standard SWP.

1.7 Cleavage of the crude product: a) Rink-Amide-Linker ${ }^{[10]}$ : The 10:90-PEGMA-co-MMA surface was incubated with $1.00 \mathrm{~mL}$ of DCM for $10 \mathrm{~min}$. Then, the DCM was removed and $1.00 \mathrm{~mL}$ of TFA/DCM/TIBS/ultrapure water $(92 \%: 3 \%: 2.5 \%: 2.5 \%(\mathrm{v} / \mathrm{v} / \mathrm{v} / \mathrm{v}))$ was pipetted onto the surface, after $15 \mathrm{~min}$ additional solution $(500 \mu \mathrm{L})$ was pipetted onto the slide to avoid drying and the surface was incubated for an additional $15 \mathrm{~min}$. Then, the surface was rinsed three times with $500 \mu \mathrm{L}$ of DCM, followed by three times with $500 \mu \mathrm{L}$ of $\mathrm{MeOH}$. The incubation and rinsing solutions were combined and the solvent was evaporated until a dry crude product was reached. b) Rink-Amide-Linker (MALDI): The 10:90-PEGMA-co-MMA surface, functionalized with a Rink-Amid-Linker, was incubated for 2 min under TFA vapor 
in a petri dish. Then, the TFA was removed for 2 min under reduced pressure. c) PhotoLinker: A 10:90-PEGMA-co-MMA surface, functionalized with a Photo-Linker, was UVirradiated with UV lamp (VL-115.L, 15 W, Vilber Lourmat GmbH, Eberhardzell, Germany) with $365 \mathrm{~nm}$ for $30 \mathrm{~min}$ (distance to source $2 \mathrm{~cm}$ ).

\subsection{Preparation of the donor slide for the laser-induced forward transfer (PDS) ${ }^{[10]}$ : A} mixture of the desired substance (amount of substance $5.72 \times 10^{-5} \mathrm{~mol}$; e.g. amino acid $\sim 15.0$ $\mathrm{mg}$ ) and resin (S-LEC-P LT 75 52; mass of resin = $150 \mathrm{mg}$ - mass of the desired substance) in $1.00 \mathrm{~mL}$ of DCM was spin-coated (spin coater by Schaefer, Langen, Germany) onto a polyimide foil covered microscope slide with $80 \mathrm{rps}$ for $40 \mathrm{~s}$.

1.9 Combinatorial laser-induced forward transfer $\left(\right.$ cLIFT) ${ }^{[10]}$ : The acceptor slide was clamped with the functionalized side facing up into the cLIFT device. The first donor surface is placed on the acceptor surface with the coated side facing down. The prepared donor slide was placed on top of the acceptor surface facing towards each other. The laser process was done as described in the literature. The irradiation periods for the spot patterns were set to: 10 $\mathrm{ms}$ for amines, $15.0 \mathrm{~ms}$ for bromoacetic acid $(\mathrm{BrAcOH}) / \mathrm{DIC}, 1.5 \mathrm{~ms}$ for bromoacetic 2,4dinitrophenylester (BrAc-ODNP), and $10 \mathrm{~ms}$ for Fmoc- $N$-substituted-glycines. The pitch was variable; functionalization of a whole surface was achieved with $5 \mathrm{~ms}$ and a pitch of $100 \mu \mathrm{m}$. The slide was heated in the oven under argon atmosphere at $90^{\circ} \mathrm{C}$ for $1 \mathrm{~h}$. After a $15 \mathrm{~min}$ cool down period, the slides were flushed extensively with DCM and washed in DCM for $3 \times 30 \mathrm{~s}$, $1 \times 30 \mathrm{~s}$ (dimethylacetamide) DMA and $1 \times 30 \mathrm{~s}$ in DCM in the ultrasonic bath to remove the matrix and unreacted chemicals. Finally, the slides were dried in a stream of argon. 
1.10 Sub-monomer method: a) Acylation: Following the PDS (see 1.8), 2.00 equivalents (equiv.) of bromoacetic acid, 2.00 equiv. of bromoacetic succinimide ester, 1.00 equiv. of BrOAc-DNP or 2.00 equiv. of bromoacetic pentafluorophenylester was spin coated with the matrix onto a donor slide, followed by the laser transfer. For the usage of inactivated bromoacetic acid, a donor slide with 4.00 equiv. of DIC was prepared and transferred, followed by the SWP. b) Nucleophilic Substitution: Following the PDS, 2.00 equivalents of amine was used to prepare the donor foil. The donor slide was dried under vacuum for $20 \mathrm{~min}$, followed by the cLIFT. Finally, the washing protocol after the oven was extended: $3 \times 3$ min DMA, $1 \times 2 \operatorname{min~} \mathrm{MeOH}$ and $1 \times 1$ min DCM.

1.11 Monomer method: 1) Following PDS, 1.00 equiv. of Fmoc- $N$-substiuted glycine, 1.00 equiv. DIC and 1.00 equiv. HOBt was spin-coated onto seperated donor slides, followed by cLIFT laser transfer. The acceptor slide was washed 5 x $30 \mathrm{~s}$ with acetone in the ultrasonic bath. Subsequently, 2) acetylation (see 1, 3) for $4 \mathrm{~h}$ and 3) Fmoc-deprotection (see 1.1) were performed.

1.12 Pre-structuring with Fmoc-Gly-OPfp: Following the PDS, $15.0 \mathrm{mg}$ of Fmoc-GlyOPfp was spin coated onto a donor slide, followed by cLIFT laser transfer (see 1.9). Subsequently, acetylation (see 1.3) and Fmoc-deprotection (see 1.1) were performed.

\subsection{Synthesis of peptoid in Figure 2A; Spot (red) and Spot (green): A 10:90-PEGMA-} co-MMA surface was pre-structured with Fmoc-Gly-OPfp (see 1.12). Following the submonomer protocol (laser duration: $1.5 \mathrm{~ms}$, pitch: $500 \mu \mathrm{m})$, BrAc-ODNP was used (1.8 1.10a). As amines, in spot (red), mono-boc-diaminobutane was used, and in spot (green) tertbutyl-aminobutanoic acid. Both amines were laser-transferred, following steps $1.8-1.10 \mathrm{~b}$. 
The fluorescence staining with Dylight 680-NHS (see 1.21) was done after the side chain deprotection with TFA (see 1.4). This was followed by an acetylation step (see 1.3), coupling of a solution of $0.2 \mathrm{M}$ mono-boc-diaminobutane, DIC and Oxyma Pure in DMF for $2 \mathrm{~h}$ (see 1.5), deprotection (see 1.1) and staining (only spot B) with TAMRA-NHS (see 1.25).

1.14 Synthesis of peptoid in Figure 2B: BrAc-ODNP was laser-transferred (irradiation period, $0.1 \mathrm{~ms}-10.0 \mathrm{~ms}$, pitch $250 \mu \mathrm{m}$ ) to an acceptor slide with free amines, following steps $1.8-1.10 \mathrm{a}$. After coupling and washing, the nucleophilic substitution was performed with aminobutyne (steps $1.8-1.10 \mathrm{~b}$ ). The fluorescence staining was done with a TAMRA-azide via click chemistry (see 1.25). The optimum irradiation period was determined via the intensities of the fluorescence signals (correlating with the yield) and the shape of the spots to achieve separate spots.

1.15 Synthesis of peptoid in Figure 2C: A 10:90-PEGMA-co-MMA surface was functionalized with the Fmoc-Rink-Amide-Linker (see 1.6). Following the submonomer protocol (laser duration: $5.0 \mathrm{~ms}$, pitch: $100 \mu \mathrm{m}$, almost completely and densely covered surface, i.e. no spots), bromoacetic 2,4-dinitrophenylester was used (steps 1.7 - 1.10). Azidopropylamine, aminobutyne, methoxyethylamine, and propargylamine (steps 1.8 1.10b) were chosen for the subsequent amination. Then, Fmoc-aminohexanoic acid was coupled with DIC, HOBt in DMF for $16 \mathrm{~h}$ (see 1.5). Finally, the crude product was cleaved with TFA from the surface (see 1.7a). MALDI: $\mathrm{C}_{44} \mathrm{H}_{56} \mathrm{~N}_{10} \mathrm{O}_{9} \mathrm{~m} / z=$ calculated: 868 ; found: $907[\mathrm{M}+\mathrm{K}]^{+}$.

1.16 Synthesis of peptoids in Figure 2D: A 10:90-PEGMA-co-MMA surface was functionalized with the Fmoc-Photo-Linker (see 1.5). Following the submonomer protocol 
(laser duration: $15 \mathrm{~ms}$, pitch: $1000 \mu \mathrm{m}$ (one spot: 4.0 shots, distance $10.0 \mu \mathrm{m}$ ), bromoaceticOSu was used (steps $1.7-1.10$ ). We used the amines aminoethanol-TBDMS, aminobutyne, methoxyethylamine, and propargylamine (steps $1.8-1.10 \mathrm{~b}$ ). Finally, we cleaved the peptoids with UV-irradiation for $30 \mathrm{~min}$ from the surface (see 1.7a) and laser-transferred the MALDImatrix material (see 2.3). Then, we incubated the surface for 2 min with TFA vapor to cleave the protecting groups.

1.17 Synthesis of peptoid in Figure 3A: PEGMA-co-MMA was functionalized, following the monomer method. Fmoc-NPhe-OH (see steps $1.8-1.9,1.11)$ was used $(100-172 \mu \mathrm{m}$, laser duration 4.0 ms). Biotin-OPfp in DMF was coupled to the free amines (see 1.5). Finally, fluorescent staining was done with Streptavidin-Atto647 (see 1.25c) in PBS-T.

\subsection{Synthesis of Peptoid in Figure 3C, Logos of IMT, IOC, IAAC: A 10:90-PEGMA-co-} MMA surface was functionalized, following the monomer method. We coupled 3 different monomers: IMT: Fmoc-Abg(Boc)-OH, IOC: Fmoc-NPhe-OH, and IAAC: Fmoc- $N$ PheOMe-OH (pitch $200 \mu \mathrm{m}$, laser duration $10 \mathrm{~ms}$, steps $1.8-1.9,1.11$ ). A solution of $0.05 \mathrm{M}$ Biotin-OPfp in DMF was given to the surface and rocked for $4 \mathrm{~h}$. The staining was done with Streptavidin-Atto647 (see 1.25c).

1.19 Synthesis of peptoid in Figure 3D: A PEGMA-co-MMA surface was functionalized with the Fmoc-Rink-Amide-Linker (see 1.6). Following the monomer protocol (laser duration: $5 \mathrm{~ms}$, pitch: $100 \mu \mathrm{m}$ ), the slide surface was nearly completely covered with the peptoid (no spots visible). We coupled the monomers Fmoc-PheCl-OH, Fmoc-NPhe-OH, and Fmoc-Abg(Boc)-OH (steps 1.8 - 1.9, 1.11 (no acetylation steps)). Then, the crude product 
was cleaved with TFA (1.7). MALDI: $\mathrm{C}_{41} \mathrm{H}_{45} \mathrm{ClN}_{6} \mathrm{O}_{6} \mathrm{~m} / z=$ calculated: 753.30; found: 754.39 $[\mathrm{M}+\mathrm{H}]^{+}$.

1.20 Synthesis of peptoid in Figure 3E: After deprotection (1.8), PEGMA-co-MMA was functionalized. Fmoc-NPhe-OH (first donor) with PyBOP (second donor), DIPEA (third donor), HOBt (fourth donor) were laser-transferred (pitch: $250 \mu \mathrm{m}$, laser duration $10 \mathrm{~ms}$ ) (1.8-1.9). After Coupling, acetylation (1.3) and deprotection (1.1), Biotin-OPfp in DMF was coupled to the free amines (see 1.5). Finally, fluorescent staining was done with Streptavidin-Atto647 (see 1.25c) in PBS-T.

1.21 Synthesis of peptoid in Figure 3F: After deprotection (1.8), PEGMA-co-MMA was functionalized. Fmoc-NPhe-OH (fourth donor), with PyBOP (first donor), DIPEA (second donor), HOBt (third donor) were laser-transferred (pitch: $250 \mu \mathrm{m}$, laser duration $10 \mathrm{~ms})(1.8$ 1.9,). After Coupling, acetylation (1.3) and deprotection (1.1), Biotin-OPfp in DMF was coupled to the free amines (see 1.5). Finally, fluorescent staining was done with Streptavidin-Atto647 (see 1.25c) in PBS-T.

1.22 Synthesis of peptoid A1: A PEGMA-co-MMA surface was functionalized with the Fmoc-Rink-Amide-Linker (see 1.6). Following the submonomer protocol (laser duration: 5 ms, pitch: $100 \mu \mathrm{m}$, surface nearly completely covered), bromoacetic acid and DIC were used (steps $1.8-1.10 \mathrm{a})$. As amines, mono-boc-diaminobutane and aminobutyne were chosen (steps $1.8-1.10 b$ ). Then, coupling of Fmoc-aminohexanoic acid with DIC, HOBt in DMF for $16 \mathrm{~h}$ was done (see 1.5). Finally, the crude product was cleaved with TFA (see 1.6). MALDI: $\mathrm{C}_{33} \mathrm{H}_{43} \mathrm{~N}_{5} \mathrm{O}_{5} \mathrm{~m} / z=$ calculated: 589.7; found: $590.3[\mathrm{M}+\mathrm{H}]^{+}, 612.3[\mathrm{M}+\mathrm{Na}]^{+}$. 
1.23 Synthesis of peptoid A2: A PEGMA-co-MMA surface was Fmoc-deprotected (see 1.1). Following the monomer protocol (DIC and HOBt in one donor, laser duration 5 ms, pitch 100 $\mu \mathrm{m}, 80 \%$ of surface covered, steps $1.8-1.11$ ), Fmoc-NPheCl-OH, Fmoc- $N$ Phe-OH, and Fmoc-Tmb-glycine-OH were used.

1.24 Synthesis of peptoid A4: A PEGMA-co-MMA surface was functionalized with the Fmoc-Rink-Amide-Linker (see 1.6). Following the submonomer protocol (laser duration $5 \mathrm{ms,}$ pitch $100 \mu \mathrm{m}$, nearly completely covered surface), active ester-DNP was transferred (steps 1.8 - 1.10a). As the amine, mono-boc-diaminobutane was chosen (steps $1.8-1.10 \mathrm{~b}$ ). This was followed by a second acylation (steps $1.8-1.10 \mathrm{a}$ ) and the nucleophilic substitution with $10 \%$ piperidine in DMF for 20 min. Then, the SWP was performed. Finally, the crude product was cleaved with TFA (see 1.6). ESI-MS: $\mathrm{C}_{15} \mathrm{H}_{29} \mathrm{~N}_{5} \mathrm{O}_{3} \mathrm{~m} / z=$ calculated: 327.4 found: 328.2 $[\mathrm{M}+\mathrm{H}]^{+}, 350.2[\mathrm{M}+\mathrm{Na}]^{+}, 365.1[\mathrm{M}+\mathrm{K}]^{+}$.

1.25 Fluorescent staining protocol/Click reaction $^{[33]}$ : a) The surface was covered with 250 $\mu \mathrm{L}$ of a solution of a 1:1 mixture of water and DMSO containing tetramethylrhodamine (TAMRA)-azide $(2.00 \mu \mathrm{g}$ per $10.0 \mathrm{~mL}), 50 \mathrm{mM}$ copper(II) sulfate and $100 \mathrm{mM}$ sodium ascorbate and a microscope slide was carefully placed on top of the surface. This sandwich was left to react overnight under argon. The surface was washed by rocking it in $1 \times 5 \mathrm{~min}$ $\mathrm{H}_{2} \mathrm{O}, 1 \times 5 \min \mathrm{DMF}, 2 \times 5 \min \mathrm{EE}, 1 \times 2 \mathrm{~min} \mathrm{MeOH}$, and $1 \times 1 \mathrm{~min} \mathrm{DCM}$, and each time the solvent was refreshed. Finally, the slides were dried under a stream of argon. b) The surface was rocked in a solution of a Fluorophor- $N$-hydroxysuccinimide (NHS) $(1.0 \mu \mathrm{g}$ per $10.0 \mathrm{~mL}$ ) in phosphate buffer saline and Tween 20 (PBS-T) for $2 \mathrm{~h}$. The surface was washed by rocking it in $1 \times 5$ min $\mathrm{H}_{2} \mathrm{O}, 1 \times 5 \mathrm{~min} D M F, 2 \times 5$ min ethylacetate $(\mathrm{EE}), 1 \times 2 \mathrm{~min}$ $\mathrm{MeOH}$ and $1 \times 1 \mathrm{~min} \mathrm{DCM}$, and each time the solvent was refreshed. Finally, the slides were 
dried under a stream of argon. c) The surface was blocked with a blocking buffer (MB-070 von Rockland, Gilbertsville, USA, for $30 \mathrm{~min}$, washed with $1 \times 1 \mathrm{~min}$ PBS-T and was rocked with a solution of Streptavidin-Atto647 in PBS-T for 2 hours (50.0 ng per $10.0 \mathrm{~mL})$ ). The surface was washed $5 \times 5$ min with PBS-T. The buffer salt was removed washing with deionized water. Finally, the slides were dried under a stream of argon.

\subsection{Synthesis of bromoacetic succinimide ester and bromoacetic}

pentafluorophenylester $^{[11,12,34]}$ : To a solution of bromoacetic acid $(1.39 \mathrm{~g}, 10 \mathrm{mmol}$, 1 equiv.) and $N$-hydroxysuccinimide $(1.15 \mathrm{~g}, 10.0 \mathrm{mmol})$ or pentafluorophenol $(1.84 \mathrm{~g}$, $10 \mathrm{mmol})$ in $50 \mathrm{~mL}$ DCM, dicyclohexylcarbodiimd (DCC) (2.06 g, $10.0 \mathrm{mmol})$ was added in three portions under strong stirring. After $2 \mathrm{~h}$, the sediment was separated and the solvent evaporated under reduced pressure. 1) bromoacetic succinimide ester was obtained as a white solid (1.83 g, $8.32 \mathrm{mmol}, 83 \%$ yield) . ${ }^{1} \mathrm{H} \mathrm{NMR}\left(500 \mathrm{MHz}, \mathrm{CDCl}_{3}, \delta\right): 4.24$ (s, 2H, Br-CH$)$. 3.00 (bs, 4H, $\mathrm{CH}_{2}-\mathrm{CH}_{2}$ ); ${ }^{13} \mathrm{CNMR}\left(126 \mathrm{MHz}, \mathrm{CDCl}_{3}, \delta\right.$ ): = 168.4 (amide- $\mathrm{C}=\mathrm{O}$ ), 163.0 (Ester-C=O), $25.3\left(\mathrm{CH}_{2}-\mathrm{CH}_{2}\right), 21.2\left(\mathrm{Br}-\mathrm{CH}_{2}\right)$. 2) bromoacetic pentafluorophenylester was obtained as a grey- brown liquid: (1.41 g, $4.62 \mathrm{mmol}, 46 \%$ yield) ${ }^{1} \mathrm{H}$ NMR (500 MHz, $\mathrm{CDCl}_{3}$, $\delta): 4.16\left(\mathrm{~s}, 2 \mathrm{H}, \mathrm{Br}-\mathrm{CH}_{2}\right) \mathrm{ppm} ;{ }^{13} \mathrm{C}-\mathrm{NMR}(126 \mathrm{MHz}, \mathrm{CDCl} 3, \delta):=163.7(\mathrm{C}=\mathrm{O}), 124.6-$ $125.1(\mathrm{~m}, \mathrm{Ar}-\mathrm{C}-\mathrm{O}), 137.2-142.2(\mathrm{~m}, \mathrm{Ar}-\mathrm{C}-\mathrm{F}), 23.9\left(\mathrm{CH}_{2}\right) \mathrm{ppm}$.

1.27 Synthesis of bromoacetic 2,4-dinitrophenylester ${ }^{[11]}$ : A solution of anhydrous 2,4dinitrophenol (3.99 g, $21.7 \mathrm{mmol}, 1.00$ equiv.) and DIPEA (3.76 mL, $21.7 \mathrm{mmol})$ in $\mathrm{CH}_{2} \mathrm{Cl}_{2}$ (30.0 mL) was cooled to $0^{\circ} \mathrm{C}$ and bromoacetylbromide $(1.80 \mathrm{~mL}, 21.7 \mathrm{mmol}, 1.00$ equiv.) in $\mathrm{CH}_{2} \mathrm{Cl}_{2}(30.0 \mathrm{~mL})$ was added dropwise. After $1 \mathrm{~h}$ at $26^{\circ} \mathrm{C}$, the organic layer was extracted with $\mathrm{H}_{2} \mathrm{O}(1 \times 30.0 \mathrm{~mL})$ and $10 \%$ citric acid in $\mathrm{H}_{2} \mathrm{O}(1 \times 30.0 \mathrm{~mL})$, dried over $\mathrm{Na}_{2} \mathrm{SO}_{4}$ and evaporated under reduced pressure to give a yellow oil. The crude product was crystallized 
from diethyl ether $(50.0 \mathrm{~mL})$ at $-18{ }^{\circ} \mathrm{C}$ to yield the active ester $(2.85 \mathrm{~g}, 9.87 \mathrm{mmol}, 45 \%$ yield $)$ as yellow crystals. ${ }^{1} \mathrm{H}$ NMR $\left(500 \mathrm{MHz}, \mathrm{CDCl}_{3}, \delta\right): 8.95\left(\mathrm{~d},{ }^{4} \mathrm{~J}=2.7 \mathrm{~Hz}, 1 \mathrm{H}, \mathrm{Ar}-\mathrm{H} 3\right), 8.51$ (dd, $\left.{ }^{3} J=8.9 \mathrm{~Hz},{ }^{4} J=2.7 \mathrm{~Hz}, 1 \mathrm{H}, \mathrm{Ar}-H 5\right), 7.49\left(\mathrm{~d},{ }^{3} J=8.9 \mathrm{~Hz}, 1 \mathrm{H}, \mathrm{Ar}-H 6\right) 4.13\left(\mathrm{~s}, 2 \mathrm{H},-\mathrm{CH}_{2}\right)$ ppm; ${ }^{13} \mathrm{C}$ NMR $\left(126 \mathrm{MHz}, \mathrm{CDCl}_{3}, \delta\right):=164.3(C=\mathrm{O}), 148.3(\mathrm{Ar}-C-\mathrm{O}), 147.2,145.5$, $129.3(\mathrm{Ar}-\mathrm{C}-\mathrm{H}), 126.4,122.0,24.3\left(\mathrm{CH}_{2}\right) \mathrm{ppm}$.

1.28 Synthesis of Fmoc-NPhe-OH ${ }^{[24]}$ : Benzylamine (12 mL, $110 \mathrm{mmol}, 2.20$ equiv.) was solved in $25.0 \mathrm{~mL}$ tetrahydrofuran (THF) and cooled to $0{ }^{\circ} \mathrm{C}$. Ethyl bromoacetate $(5.54 \mathrm{~mL}$, $50.0 \mathrm{mmol}$ ) in $25 \mathrm{~mL}$ THF was given dropwise to the stirring solution and, then, was stirred for $2.5 \mathrm{~h}$ at room temperature. THF was removed under reduced pressure. The crude product was purified via column chromatography with diethyl ether. $4.0 \mathrm{M} \mathrm{NaOH}(9.10 \mathrm{~mL})$ was added to a solution of $\mathrm{H}-N \mathrm{Phe}-\mathrm{OEt}(7.04 \mathrm{~g}, 36.5 \mathrm{mmol})$ in dioxane $(128 \mathrm{~mL})$ and $\mathrm{MeOH}$ (46 mL). After stirring for $30 \mathrm{~min}$ at room temperature, the reaction mixture was concentrated in vacuo to give $\mathrm{H}-\mathrm{NPhe}-\mathrm{ONa}$. The sodium salt was dissolved in water $(36.5 \mathrm{~mL})$ and the $\mathrm{pH}$ adjusted to $9-9.5$ with concentrated hydrochloric acid. To this mixture, a solution of FmocOSu (12.29 g, $36.5 \mathrm{mmol}, 1.00$ equiv.) in acetonitrile $(73.0 \mathrm{~mL})$ was added in one portion. Stirring was continued for 30 minutes, and the $\mathrm{pH}$ was maintained at $\mathrm{pH} 8.5-9.0$ by the addition of triethylamine (TEA). Acetonitrile was removed under reduced pressure and the residue was poured into $20 \%$ citric acid $(219 \mathrm{~mL})$. The aqueous layer was extracted with EtOAc $(4 \times 60 \mathrm{~mL})$, and the combined organic layers were washed with water and brine, dried over $\mathrm{Na}_{2} \mathrm{SO}_{4}$, and concentrated in vacuo to give Fmoc- $N \mathrm{Phe}-\mathrm{OH}$ as oil which was crystallized from EtOAc / hexanes to afford Fmoc- $N$ Phe-OH $(4.66 \mathrm{~g}, 12.03 \mathrm{mmol})$ as a white solid in $24 \%$ yield (over 3 steps). In the NMR spectra both rotamers are present; ${ }^{1} \mathrm{H}$ NMR (500 MHz, $\mathrm{CDCl}_{3}, \delta$ ): $7.76\left(\mathrm{~d}, 2 \mathrm{H},{ }^{3} J=7.5 \mathrm{~Hz}, \mathrm{Ar} H \mathrm{Fmoc}\right.$ ), 7.58, 7.53 (two d, ${ }^{3} J=7.4 \mathrm{~Hz}, 2 \mathrm{H}$, ArH-Fmoc), 7.20 -7.43 (m, 8H, ArH-Fmoc, Ph), 7.09 - 7.19 (m, 1H, Ph), 4.59 (d, 2H, $\mathrm{CH}_{2^{-}}$ 
Fmoc, $J=6.3 \mathrm{~Hz}$ ) 4.53, 4.59 (two s, $2 \mathrm{H}, \mathrm{CH}_{2} \mathrm{Ph}$ ), $4.25-4.31$ (m, 1H, CH-Fmoc), 3.79, 4.03

(two s, 2H,NCH${ }_{2} \mathrm{C}(\mathrm{O})$ ) ppm; ${ }^{13} \mathrm{C}$ NMR $\left(126 \mathrm{MHz}, \mathrm{CDCl}_{3}, \delta\right): 175.1\left(\mathrm{NCH}_{2} \mathrm{C}(\mathrm{O}) \mathrm{OH}\right) .156 .9$

(Fmoc CO), 156.6, 144.1 (ArC-Fmoc), 141.6, $2 \times 136.4,2 \times 129.0,128.4,2 \times 128.0,127.8$, $2 \times 127.3, \quad 125.1$ (125.0), 120.2, $68.3\left(\mathrm{CH}_{2}-\mathrm{Fmoc}\right), \quad 67.9, \quad 51.6, \quad 51.3\left(\mathrm{CH}_{2} \mathrm{Ph}\right), 48.0$ $\left(\mathrm{NCH}_{2} \mathrm{C}(\mathrm{O})\right), 47.3$ (47.4) (CH-Fmoc), $47.0\left(\mathrm{NCH}_{2} \mathrm{C}(\mathrm{O})\right)$ ppm; HRMS $\left(\mathrm{C}_{24} \mathrm{H}_{21} \mathrm{O}_{4} \mathrm{~N}_{1}\right)$ : calcd. 387.1465, found 387.1466 .

1.29 Synthesis of Fmoc-NPheCl-OH ${ }^{[24]}$ : 4-Chlorobenzylamine $(8.00 \mathrm{~mL}, 66.0 \mathrm{mmol})$ was dissolved in $15 \mathrm{~mL}$ THF and cooled to $0{ }^{\circ} \mathrm{C}$. Ethyl bromoacetate $(3.32 \mathrm{~mL}, 30 \mathrm{mmol}, 1.00$ equiv.) in $25.0 \mathrm{~mL}$ THF was added dropwise to the stirring solution, which was then, further stirred for $2.5 \mathrm{~h}$ at room temperature. THF was removed under reduced pressure. The crude product was purified via column chromatography with diethyl ether. $4 \mathrm{M} \mathrm{NaOH}(3.70 \mathrm{~mL})$ was added to a solution of $\mathrm{H}-\mathrm{NPheCl}-\mathrm{OEt}(3.36 \mathrm{~g}, 14.8 \mathrm{mmol})$ in dioxane $(520 . \mathrm{mL})$ and $\mathrm{MeOH}(18.5 \mathrm{~mL})$. After stirring for $30 \mathrm{~min}$ at room temperature, the reaction mixture was concentrated in vacuo to give H-NPhe-ONa. The sodium salt was dissolved in water (14.8 $\mathrm{mL}$ ) and the $\mathrm{pH}$ adjusted to $9-9.5$ with concentrated hydrochloric acid. To this mixture, a solution of Fmoc-OSu (4.99 g, $14.8 \mathrm{mmol})$ in acetonitrile $(30.0 \mathrm{~mL})$ was added in one portion. Stirring was continued for 30 minutes, and the $\mathrm{pH}$ was maintained at $\mathrm{pH} 8.5-9.0$ by the addition of TEA. Acetonitrile was removed under reduced pressure and the residue was poured into $20 \%$ citric acid $(89.0 \mathrm{~mL})$. The aqueous layer was extracted with EtOAc $(3 \times 60$ $\mathrm{mL}$ ), and the combined organic layers were washed with water and brine, dried over $\mathrm{Na}_{2} \mathrm{SO}_{4}$, and concentrated in vacuo to give Fmoc-NPheCl-OH as an oil which was crystallized from EtOAc/hexanes to afford Fmoc- $N \mathrm{PheCl}-\mathrm{OH}(1.40 \mathrm{~g}, 3.32 \mathrm{mmol})$ as a white solid in $11 \%$ yield (over 3 steps). In the NMR spectra both rotamers are present; ${ }^{1} \mathrm{H} \mathrm{NMR}\left(500 \mathrm{MHz}, \mathrm{CDCl}_{3}, \delta\right)$ : $7.78-7.75$ (m, 2H, ArHFmoc), 7.57, 7.47 (two d, ${ }^{3} J=7.4 \mathrm{~Hz}, 2 \mathrm{H}, \mathrm{Ar} H-\mathrm{Fmoc}$ ), $7.45-7.13$ 
(m, 8H, ArH-Fmoc, Ph), $6.95-6.93$ (m, 1H, Ph), 4.63, 4.60 (two d, 2H, $\mathrm{CH}_{2}-\mathrm{Fmoc},{ }^{3} J=6.1$

$\mathrm{Hz}$ ), 4.53, 4.38 (two s, 2H, $\mathrm{CH}_{2} \mathrm{Ph}$ ), 4.28 - 4.24 (m, 1H, CH-Fmoc), 3.99, 3.76 (two s,

2H,NCH$\left.H_{2} \mathrm{C}(\mathrm{O})\right)$ ppm; ${ }^{13} \mathrm{C}$ NMR $\left(126 \mathrm{MHz}, \mathrm{CDCl}_{3}, \delta\right): 174.5\left(\mathrm{NCH}_{2} \mathrm{C}(\mathrm{O}) \mathrm{OH}\right) .156 .8$ (Fmoc

CO), 156.6, 144.9 (ArC-Fmoc), 141.6, 2 x 135.2, $2 \times 133.9,129.7,129.1,127.9,127.3,125.0$,

120.3, $68.0\left(\mathrm{CH}_{2}-\mathrm{Fmoc}\right), 51.1,51.0\left(\mathrm{CH}_{2} \mathrm{Ph}\right), 48.2\left(\mathrm{NCH}_{2} \mathrm{C}(\mathrm{O})\right), 47.3$ (47.5) (CH-Fmoc),

$47.2\left(\mathrm{NCH}_{2} \mathrm{C}(\mathrm{O})\right)$ ppm; HRMS $\left(\mathrm{C}_{24} \mathrm{H}_{20} \mathrm{O}_{4} \mathrm{~N}_{1} \mathrm{Cl}_{1}\right)$ : calc. 421.1075, found 421.1077.

\section{Synthesis of the arrays}

A
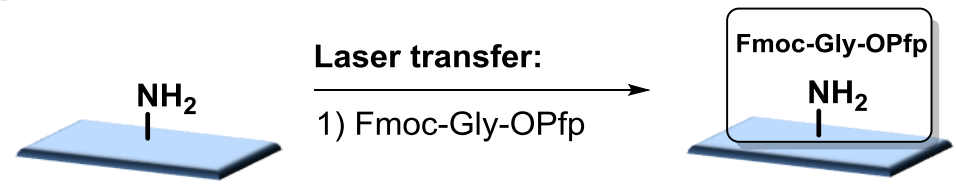

B
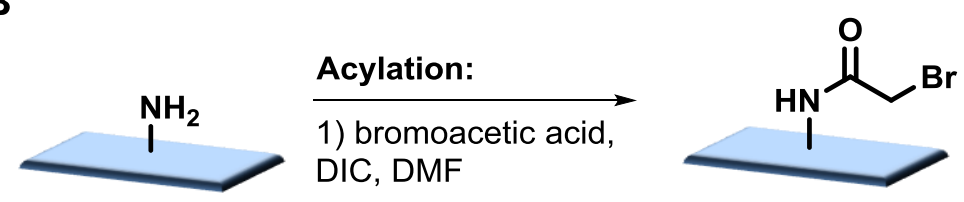
Substitution:

2) $\mathrm{NH}_{2}-\mathrm{R}, \mathrm{DMF}$
Nucleophilic
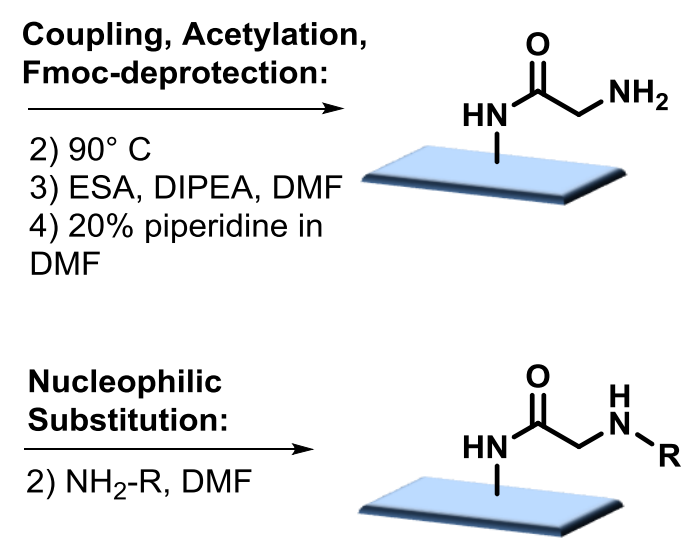

C Amide bond formation:

1) Fmoc-N-glycine-OH, PyBOP, DIPEA, DMF

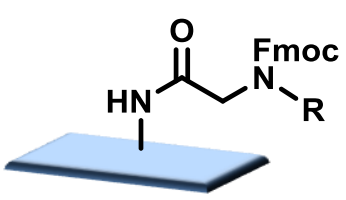

Fmoc-deprotection:

2) $20 \%$ piperidine in DMF

Figure S1: Synthesis of molecule arrays. (A) Peptide synthesis via the laser-based approach. 1) Laser transfer of the amino acid e.g. Fluorenylmethyloxycarbonyl-glycine-pentafluorophenylester (Fmoc-Gly-OPfp) 2) Coupling in the oven $\left(90^{\circ}\right.$ C) 3) Acetylation via acetic anhydride (ESA), N,N-diisopropylethylamine (DIPEA) in dimethylformamide (DMF) 4) Fmoc-deprotection via 20\% piperidine in DMF. (B) Submonomer method 1) $N, N^{\prime}$-diisopropylcarbodiimide (DIC) and bromoacetic acid in DMF 2) Nucleophilic substituation via amine in DMF. (C) Monomer method 1) Fmoc- $N$-glycine-OH, benzotriazol-1-yl-oxytripyrrolidinphsphonium hexafluorophosphate PyBOP, DIPEA in DMF 2) Fmoc-deprotection via 20\% piperidine in DMF. 


\section{Results}

\section{1 (MALDI) mass spectrometry of peptoids (crude product)}

PEGMA-co-MMA surfaces (on glass microscopy slides standard US format) were derivatized with a Fmoc-Rink-Amide-Linker. Fmoc-Gly-OPfp, chosen as spacer, was coupled to the free amine after acetylation and deprotection with $20 \%$ piperidine in DMF. The peptoid was built up layer-by-layer with the laser-based approach on the entire surface (overlapping spots), following the sub-monomer protocol (Figure S2) (see, 1.22) or the monomer protocol (see $1.21)$.

A

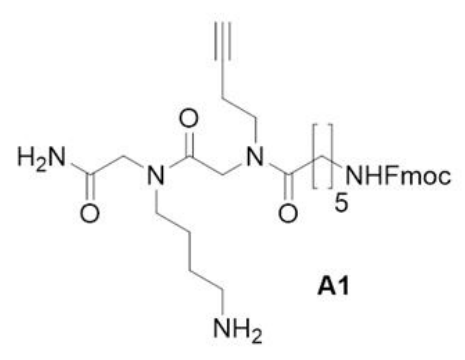

B

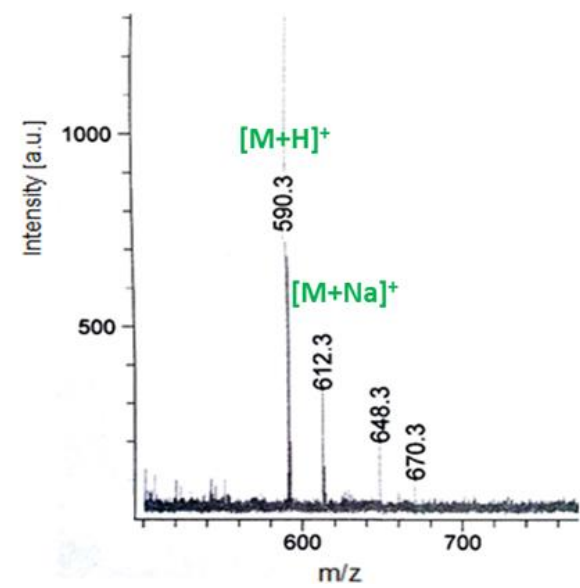

Figure S2 Example of the detected peptoid A1 via standard protocol. (A) Acylation: BrAcOH, DIC, $\mathrm{S}_{\mathrm{N}}$ 2: mono-Boc-diaminobutane/Acylation: $\mathrm{BrAcOH}, \mathrm{DIC}, \mathrm{S}_{N} 2$ : aminobutyne; coupling of Fmoc-aminohexanoic acid with DIC, HOBt in DMF; cleavage of the crude product with TFA; (matrix: 2.5-dihydroxybenzoic acid; dissolved in acetonitrile/ 0.1\% TFA in water (30:70 vol-\%). (B) Mass spectrum of product A1. 
The crude product was cleaved via TFA, TIBS, $\mathrm{H}_{2} \mathrm{O}$ in DCM and the solution was removed under vacuum (see 1.7a). The peptoids were analysed with MALDI-MS.
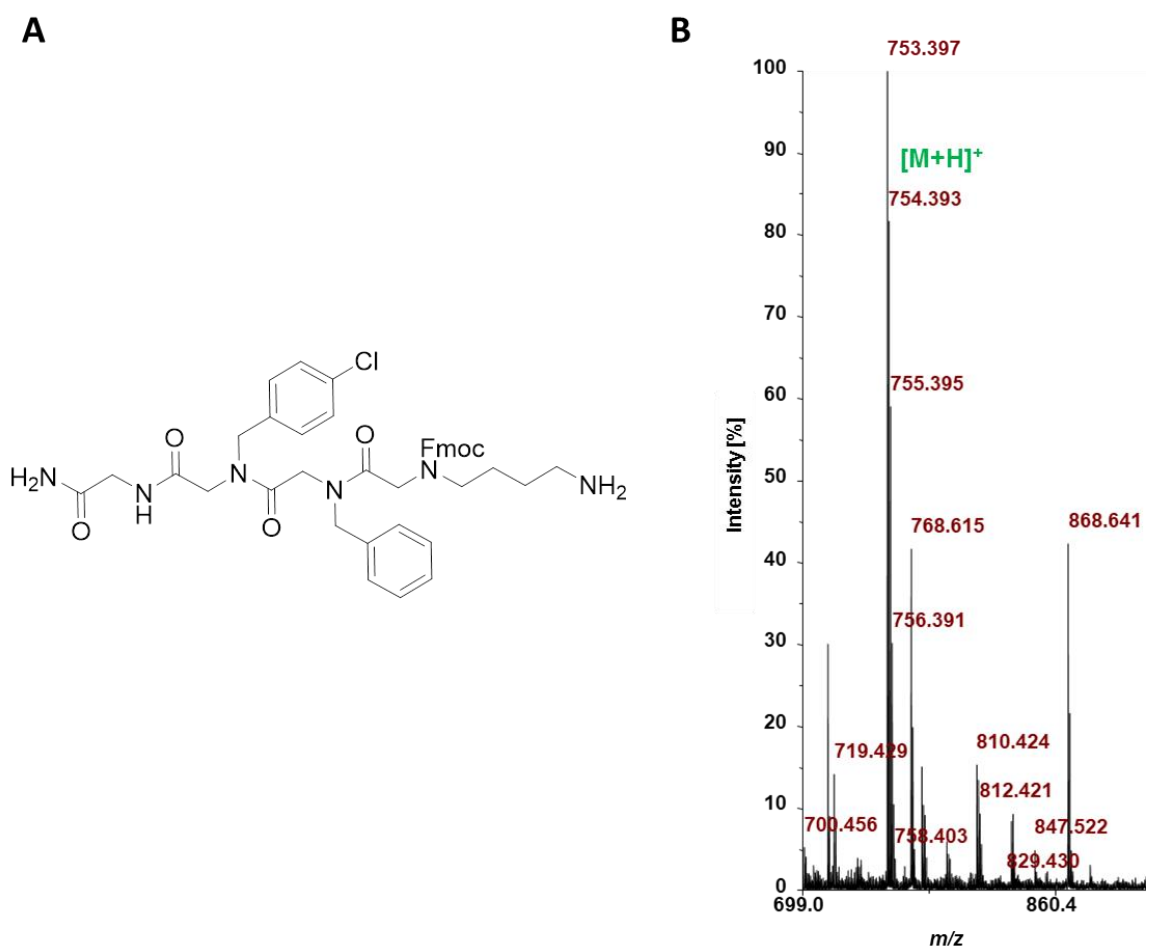

Figure S3: (A) Detected peptoid (Figure 2C) via standard protocol (monomer method: Fmoc-NPheCl-OH, Fmoc- $N$ Phe-OH and Fmoc-Abg(Boc)-OH); (matrix: DHB/CHCA MALDI matrix mixture was dissolved in acetonitrile/0.1\% TFA in water (50:50 vol\%). (B) Mass spectrum of the peptoid (Figure 2C).

\subsection{Optimization}

\subsubsection{Acylation}

Optimization of the acylation yield with BrAcOH, DIC:

a) Donor system: Three different approaches with different layers were used on one slide in three different areas. Area 1: $\mathrm{BrAcOH}$ (layer 1), DIC (layer 2); Area 2: DIC (layer 1), BrAcOH (layer 2), DIC (layer 3); Area 3: DIC (layer 1), BrOAc (layer 2) 
were laser-transferred to a PEGMA-co-MMA surface with free amines. After coupling in the oven, a donor slide was prepared with an amine (aminobutyne) for the nucleophilic substitution (steps $1.8-1.10$ ). The amine was laser-transferred to all three areas and coupled. The fluorescence staining was done with TAMRA-azide, $\mathrm{CuSO}_{4}$, sodium ascorbate, DMSO and $\mathrm{H}_{2} \mathrm{O}$ for $24 \mathrm{~h}$ (see 1.23b). The coupling efficiency and the spot shape were investigated via fluorescence intensity. The highest intensity was detected with $\mathrm{BrAcOH} / \mathrm{DIC}$ and $\mathrm{DIC} / \mathrm{BrAcOH} / \mathrm{DIC}$. However, the system $\mathrm{BrAcOH} / \mathrm{DIC}$ should be avoided, because of the $\mathrm{N}$-alkylation (inactivated BrOAc can react with free amines).

b) Irradiation period: The coupling efficiency of the acylation depends on the amount of available substances, transferred to the surface. Increased irradiation periods lead to more transferred material. The irradiation period is limited, because too long irradiations (1) cause destruction of the polymer synthesis surface or (2) cause spots to blend into each other and overlap. Therefore, irradiation periods between $100 \mu \mathrm{s}-20$ ms were tested. DIC (layer 1), BrAcOH (layer 2), DIC (layer 3) were laser-transferred to a PEGMA-co-MMA surface with free amines. After coupling in the oven for the nucleophilic substitution, one donor was prepared with an amine (aminobutyne). The amine was laser-transferred using different irradiation periods and, afterwards, coupled. The fluorescence staining was done with TAMRA-azide, $\mathrm{CuSO}_{4}$, sodium ascorbate, DMSO and $\mathrm{H}_{2} \mathrm{O}$ for $24 \mathrm{~h}$ (steps $1.8-1.10$ ). The coupling efficiency and the spot shape were investigated via fluorescence analysis. Irradiation periods of $15 \mathrm{~ms}$ gave the best results. Higher intensities destroyed the surface and should be avoided.

\section{$\underline{\text { Usage of active ester: }}$}


The usage of an alternative acylation reagent was investigated, because DIC is watersensitive and the strong laser irradiation might lead to decomposition. One donor of an active ester would replace the three donor system of DIC/BrAcOH/DIC.

c) Test of yields with different active esters: The active esters, namely bromoacetic succinimide ester (OSu), bromoacetic 2,4-dinitrophenylester (ODNP), and bromoacetic pentafluorophenylester (OPfp) were laser-transferred in a chessboard pattern with 2 areas $\mathrm{AB}$ and $\mathrm{CD}$ (irradiation period $15 \mathrm{~ms}$ for highest material transfer, laser power 100\%). After coupling and washing, the nucleophilic reaction was done with aminobutyne (steps $1.8-1.10)$. The fluorescence staining was performed with tetramethylrhodamine 5-carboxamido-6-azidohexanyl (TAMRA-azide) (see 1.25b). The fluorescence signal correlates with the yield and should show the efficiency of the reaction. In the literature, ${ }^{[11]}$ the active ester-DNP and -OPfp gives high yields, side reactions and terminations were not found. The active ester-OSU led to lower yields and as a side reaction $N$-alkylation occured (see. Fig. S3). In our laser-based approach, it was important to investigate the coupling yields. Surprisingly, the highest fluorescence signal was reached with bromoacetic succinimide ester followed by bromoacetic 2,4-dinitrophenylester with similar intensities and bromoacetic pentafluorophenylester with very weak fluorescence signals (possibly instable because of liquid state). However, we decided to avoid bromoacetic succinimide ester for the acylation, because of a possible $N$-alkylation described in the literature. ${ }^{[11]}$ The active ester-DNP, not causing side reactions, ${ }^{[11]}$ was used for further experiments.

d) Irradiation period: Irradiation periods between $100 \mu \mathrm{s}-10.0 \mathrm{~ms}$ were tested. Bromoacetic acid-DNP was laser-transferred to a PEGMA-co-MMA surface with free amines. After coupling in the oven for the nucleophilic substitution, one donor was prepared with an amine (aminobutyne) (steps $1.8-1.10$ ). The amine was laser- 
transferred and coupled. The fluorescence staining was done with TAMRA-azide, $\mathrm{CuSO}_{4} \times 5 \mathrm{H}_{2} \mathrm{O}$, Sodium ascorbate, DMSO and $\mathrm{H}_{2} \mathrm{O}$ (see 1.25b). The yield and the spot shape were investigated via the fluorescence analysis. Good intensities were achieved with a irradiation period of $1.50 \mathrm{~ms}$, increased irradiation period leads to a higher coupling yield. However, the spot size increased with the irradiation period, limiting the approach to $1.5 \mathrm{~ms}$ irradiation periods for separate spots (Figure 2B).

\subsubsection{Nucleophilic substitution}

Optimization of the yield:

a) Irradiation period: Irradiation periods between $100 \mu \mathrm{s}-20 \mathrm{~ms}$ were tested in different experiments (see 3.2.1 acylation). For separate spots with good yields, an irradiation time of $10 \mathrm{~ms}$ should be used. Irradiation periods $>15 \mathrm{~ms}$ resulted in partial destruction of the surface.

b) Optimization of the contamination: Contamination of the acceptor slide with the amines from the donor slides was detected, only due to the contact of donor and acceptor slide. Therefore, the donor slides were dried for $15 \mathrm{~min}$ in a desiccator under vacuum, to completely remove the solvent, which efficiently reduced the contamination. Furthermore, the surface was pre-structured with an amino acid with the laser-transfer method, which completely prevented contamination. Fmoc-GlyOPfp was laser-transferred to a PEGMA-co-MMA surface with a free amine in a chessboard pattern $\mathrm{AB}$ (pitch $500 \mu \mathrm{m}$ ). Bromoacetic 2,4-dinitrophenylester was lasertransferred (intensity: $100 \%$, irradiation period: $1.50 \mathrm{~ms}$ ) in the cheeseboard pattern AB. After coupling in the oven for the the nucleophilic substitution the amine (monoBoc-diaminobutane) and amine (tert-butyl-amino-butanoic acid) were used. The donor slides were dried under vacuum for $15 \mathrm{~min}$. On position A mono-boc-diaminobutane 
was laser-transferred, on position B tert-butyl-amino-butanoic acid $(1.8-1.10)$. The surface was acetylated two times (1.2). The side chain-protecting groups were removed via TFA, TIBS, $\mathrm{H}_{2} \mathrm{O}$, DCM (1.4). The fluorescence staining was done with DyLight 650 NHS in PBS-T (1.25b). A positive signal of spot $\mathbf{B}$ after fluorescence detection would show a contamination during the laser process (Figure S4). Only the spot A shows fluorescence, therefore a contamination can be excluded.

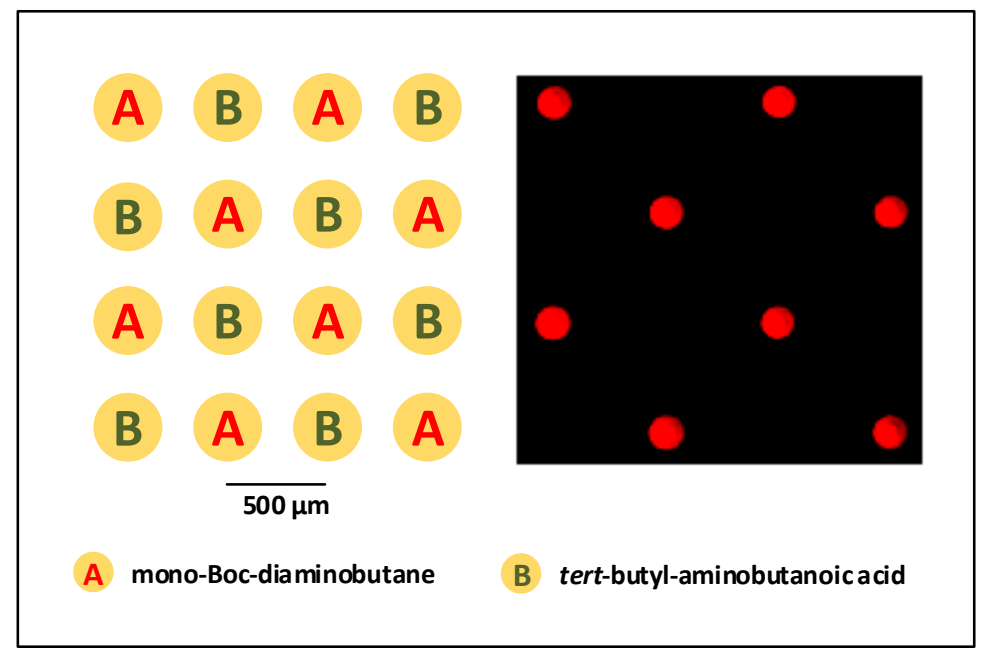

Figure S4 Chessboard after the coupling of two different amines: Spot A mono-bocdiaminobutane and Spot B tert-butyl-aminohexanoic acid. Only Spot A shows a fluorescent signal, no contamination occurs.

\subsubsection{Laser-based approach}

a) ESI mass spectrometry: To evaluate our laser-based synthesis approach of peptoids, we synthesized a 2-mer peptoid A4 (Scheme S1) with active ester-DNP and two amines (cLIFT: mono-boc-diaminobutane and in solution piperidine in DMF) on a Rink-AmideLinker surface, bearing a glycine with free amines. After cleavage of the crude product, mass 
spectrometry (solvent: $50 \%$ acetonitrile/ $0.1 \%$ formic acid in water) was carried out to identify truncated sequences or side reactions.

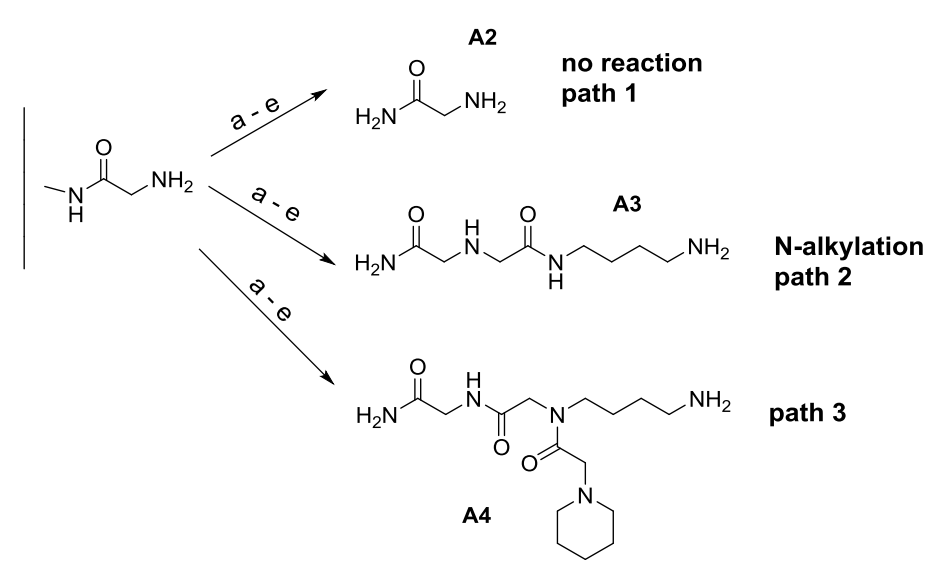

Scheme S1 Possible reactions on the surface: a) cLIFT: Acylation with active ester-DNP, b) cLIFT: Nucleophilic substitution with mono-Boc-diaminobutane c) cLIFT: Acylation with active ester-DNP, d) Nucleopilic substitution with piperidine in DMF, e) Cleavage with TFA; Path 1: no reaction (termination). Path 2: $\mathrm{N}$-alkylation instead of acylation (side reaction). Path 3: Acylation.

The product resulted in the strongest signals. In the mass spectrum (Figure S5), the mass peak of the termination product $\mathbf{A} \mathbf{2}$ in path 1 was found. The first acylation did not saturate the free amines on the surface. This result was also reported with the laser-based synthesis of peptide arrays. ${ }^{[10]}$ The truncation problem can be solved by repeating the laser process (transfer and coupling). Only very weak mass peaks of the side products ( $N$-alkylation: A3) were detected. 


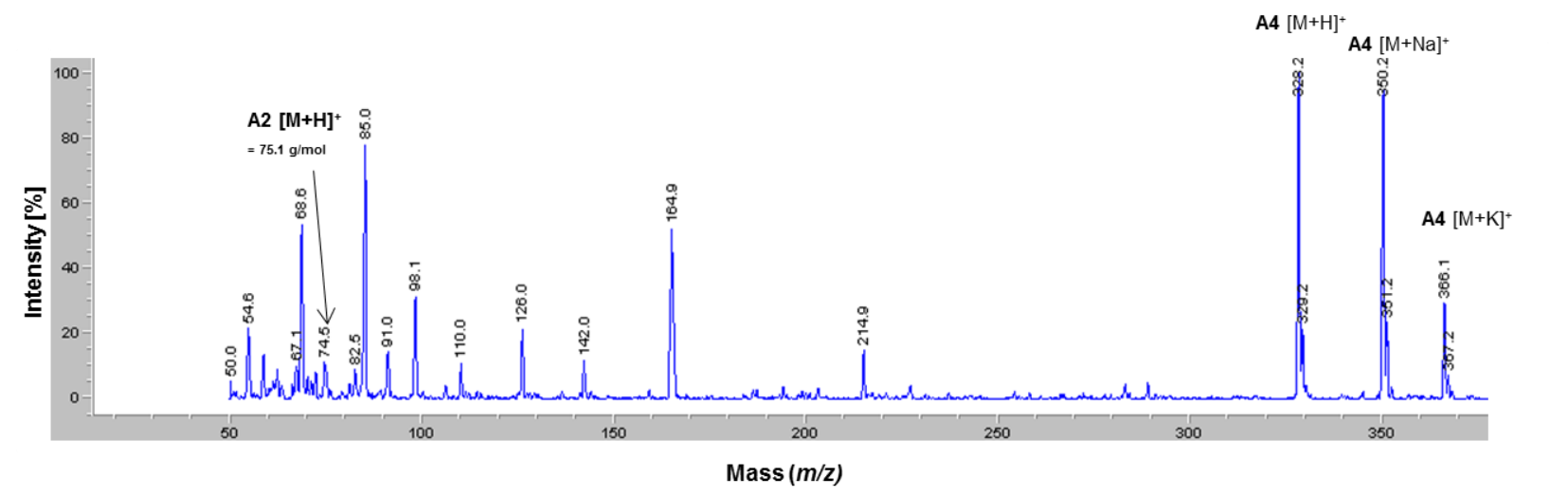

Figure S5 Mass spectrum: Detection of the mass peak of path 1 (no reaction), $\mathbf{A 2}$ and the product A4 (see Scheme S1).

\subsection{Laterally resolved MALDI-MS of peptoids}

PEGMA-co-MMA microscope surfaces were derivatized with a Fmoc-Photo-Linker (see 1.5), or as an alternative, Fmoc-Rink-Amide-Linker can be used (see 1.6). Fmoc-Gly-OPfp was coupled as a spacer to the free amine after acetylation and deprotection with $20 \%$ piperidine in DMF (see 1.5). The peptoid was synthesized layer-by-layer with the laser-based method with the desired pattern (steps $1.8-1.10$ ). Finally, a Fmoc-amino acid was coupled to achieve a higher molecular mass. The side chain protecting groups were cleaved via TFA. The crude product was cleaved via UV-irradiation (UV-lamp (VL-115.L, 15 W, Vilber Lourmat GmbH, Eberhardzell, Germany). (Rink-Amide-Linker: Cleavage of the molecules with TFA vapor, see 1.7). Afterwards, the MALDI matrix (1:1 mixture of 2,5-dihydroxybenzoic acid (DHB)/ $\alpha$ cyano-4-hydroxycinnamic acid (CHCA)) was transferred via cLIFT, corresponding to the peptoid spot pattern (example: Figure S6B). This approach avoids diffusion and results in patterned matrix layers without obscuring the microscopic visibility of peptoid spots. The preparation of a donor slide with MALDI-matrix was done as follows: $150 \mathrm{mg}$ MALDI 
DHB/CHCA matrix mixture was dissolved in $2.0 \mathrm{~mL} \mathrm{MeOH}$ and spin-coated onto a polyimide foil covered microscope slide with $50 \mathrm{rps}$ for $10 \mathrm{~s}$, followed by $80 \mathrm{rps}$ for $30 \mathrm{~s}$. The cleaved peptoids on the surface were detected via a MALDI-MS line scan. In a first experiment 8 different amines were used to build up a peptoid array (Figure S6).

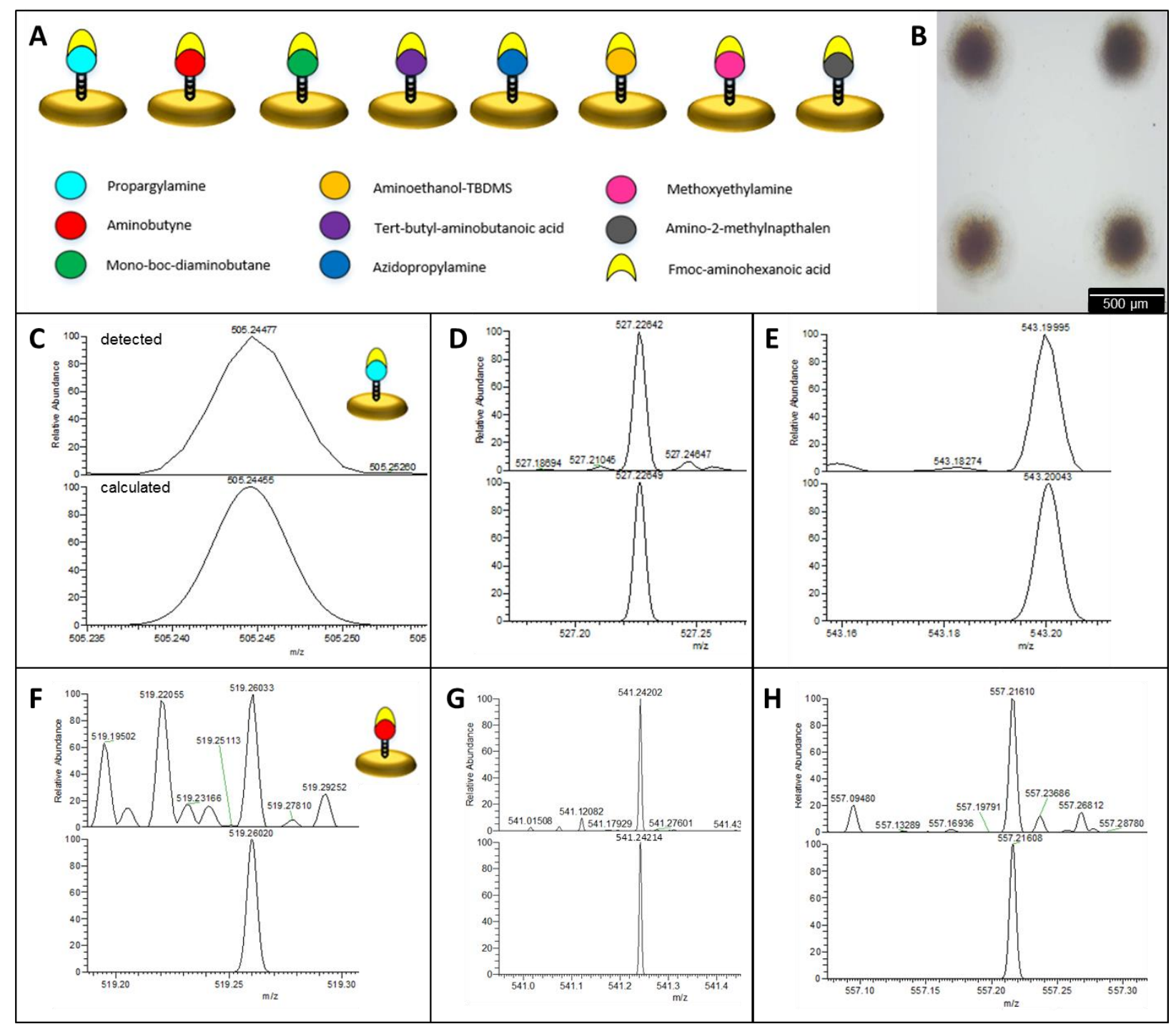

Figure S6 (A) Assembly of the peptoids, detected via a AP-MALDI Orbitrap MS line scan. (B) Light microscope image of the laser-transferred DHB/CHCA MALDI matrix. (C) Example of a laterally resolved mass spectrum of a peptoid with BrOAc/DIC and propargylamine. MALDI: $\mathrm{C}_{28} \mathrm{H}_{32} \mathrm{~N}_{4} \mathrm{O}_{5} ;[\mathrm{M}+\mathrm{H}]^{+} \mathrm{m} / z=505.24455$ (calculated); 505.24477 
(detected), (D) $[\mathrm{M}+\mathrm{Na}]^{+} \mathrm{m} / \mathrm{z} 527.22649$ (calculated); 527.22642 (detected), (E) $[\mathrm{M}+\mathrm{K}]^{+} \mathrm{m} / \mathrm{z}=$ 543.20043 (calculated); 543.1995 (detected). (F) Example of a laterally confined mass spectrum of a peptoid with BrAcOH/DIC and aminobutyne. MALDI: $\mathrm{C}_{29} \mathrm{H}_{34} \mathrm{~N}_{4} \mathrm{O}_{5}$; $[\mathrm{M}+\mathrm{H}]^{+}$ $m / z=519.26020$ (calculated); 519.26033 (detected). (G) $[\mathrm{M}+\mathrm{Na}]^{+} \mathrm{m} / z=541.22020$ (calculated), 541.24214 (detected), $(\mathrm{H})[\mathrm{M}+\mathrm{K}]^{+} \mathrm{m} / \mathrm{z}=557.21606$ (calculated); 557.21610 (detected

(Laser duration: $5 \mathrm{~ms}$, pitch $100 \mu \mathrm{m}, 8$ blocks). Only a line scan on the surface of 8 peptoid blocks was done to show, whether the peptoids were built up and the laser-transfer of the amines is possible. In Figure S5 the mass spectra of two peptoids are shown.

\subsection{Pitch experiment with the monomer method}

To study, which spot distances (pitches) are possible, maintaining separate spots, Fmoc- $N$ Phe$\mathrm{OH}$ (first layer was coupled via the monomer approach (steps $1.8-1.9,1.11$, pattern see Figure S7) was coupled. For the staining, biotin-OPfp was coupled (see 1.5) and then incubated with Streptavidin (see 1.23c) (Figure 3).

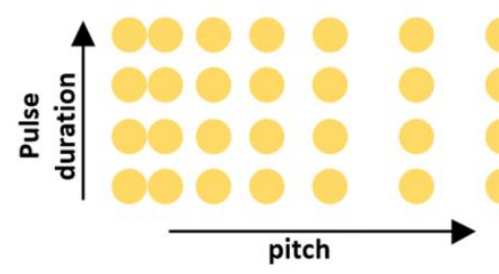

Figure S6: Layout with an increased pitch $(100 \mu \mathrm{m}-1.00 \mathrm{~mm})$ and an increased irradiation period (1.00 ms 10.0 ms) 


\section{Materials}

Chemicals and solvents for the synthesis were used from abcr (Karlsruhe/Deutschland), Iris Biotech (Marktredwitz/Germany), ThermoFisher Scientific (Waltham/USA), Alfa Aesar (Karlsruhe/Germany), Merck (Darmstadt/Germany), Sigma Aldrich Chemie GmbH (Steinheim/Germany), VWR (Darmstadt/Germany) and used for the synthesis without purification. DCM (dry) and DMF (dry) were stored above $4 \AA$ molar sieve. The Fmoc- $N$ substituted glycines Fmoc- $N$-(4-Boc-aminobutyl)-glycine (Fmoc-Abg(Boc)-OH), Fmoc- $N$ (2,4,6-trimethoxybenzyl)-glycine (Fmoc-TmbGly-OH) were obtained from Iris Biotech (Marktredwitz/Germany). Mono-boc-diaminobutane, tert-butyl-aminobutanoic acid, azidopropylamine and tert-butyl-dimethylsilyl (TBDMS)-aminoethanol were obtained from Anne Schneider (KIT/Germany). The DHB/CHCA MALDI matrix mixture was obtained from Sigma Aldrich Chemie GmbH ("universal matrix”, Steinheim/Germany). Polyimide Kapton foil was bought from CMC Klebetechnik GmbH (Frankenthal/Germany) and the resin (S-LEC-P LT 7552) from Sekisui Chemical Co. Ltd. (Osaka/Japan). The synthesis surfaces (10:90 poly(ethylene glycol) methacrylate-co-methyl methacrylate (PEGMA-co-MMA), functionalized with one Fmoc- $\beta$-Ala; were obtained from PEPperPRINT GmbH (Heidelberg/Germany). Ultrapure water was produced with a Synergy ${ }^{\circledR}$ Water Purification System.

\section{Analytical methods}

ESI-MS was measured on an HP/Agilent 1100 series LC-MS equipped with a mass selective detector (G1946A) and a diode array detector (G1315A) using an eluent composition of 50\% acetonitrile in water with $0.1 \%$ formic acid and a flow rate of $1 \mathrm{ml} / \mathrm{min} .{ }^{1} \mathrm{H}-\mathrm{NMR}$ spectra and

${ }^{13} \mathrm{C}-\mathrm{NMR}$ spectra were measured on a Bruker Avance 500 from the Bruker Corporation (Billerica/USA) at $500 \mathrm{MHz}$ and $126 \mathrm{MHz}$. Chemical shifts are expressed in parts per million 
(ppm, $\delta$ ) downfield from tetramethylsilane (TMS) and are referenced to the residual protons of $\mathrm{CDCl}_{3}(7.26 \mathrm{ppm})$ which was used as solvent. All coupling constants are absolute values and $J$ values are expressed in Hertz $(\mathrm{Hz})$. For assigning signal separation of ${ }^{1} \mathrm{H}$ NMR spectra the following abbreviations were used: $\mathrm{s}=$ singlet, $\mathrm{bs}=$ broad singlet, $\mathrm{d}=$ doublet, $\mathrm{t}=$ triplet, $\mathrm{m}=$ multiplet, $\mathrm{dd}=$ doublet of doublets. EI-MS (electron ionization mass spectrometry) was performed by using a Finnigan MAT 90 (70 eV). Matrix-assisted Laser Desorption/Ionization Time of Flight (MALDI-ToF) measurements were performed at the DKFZ in Heidelberg on an Ultraflex ${ }^{\mathrm{TM}}$ TOF I instrument (Bruker Daltonik, Bremen, Germany) equipped with a nitrogen laser. The instrument was operated with positive-ion reflecton mode, ion source voltage 1 (ion acceleration voltage) $25.0 \mathrm{kV}$, ion source voltage 2 (first extraction plate) 21.9 $\mathrm{kV}$, ion source lens voltage $6 \mathrm{kV}$ and reflectron voltage $26.3 \mathrm{kV}$, or were performed at the KIT in Karlsruhe (IFG) on a 4800 MALDI-ToF/ToF mass spectrometer (Applied Biosystems/MDS SCIEX, Foster City, CA) equipped with a pulsed Nd:YAG laser (355 nm wavelength with $<500$ ps pulses and $200 \mathrm{~Hz}$ repetition rate) and the 4000 Series Explorer (V 3.5.3) and Data Explorer (V 4.9) software. Data acquisition was performed in the reflector positive ion mode. As the matrix, 2.5-dihydroxybenzoic acid (Bruker, Daltonik) or DHB/CHCA MALDI matrix mixture was dissolved in acetonitrile/ 0.1\% TFA in water (30:70 vol\%) or acetonitrile/0.1\% TFA in water (50:50 vol\%) and placed on a ground steel target. A peptide calibration standard II from Bruker Daltonik was used. The used software was FlexControl version 2.4 for instrument control and FlexAnalysis version 2.4 for spectrum processing. $[\mathrm{M}+\mathrm{H}]^{+}$is the protonated molecule. A high-resolution atmospheric pressure imaging ion source (AP-SMALDI10, TransMIT GmbH, Giessen, Germany) coupled to an orbital trapping mass spectrometer (Q Exactive, Thermo Fisher Scientific GmbH, Bremen, Germany) was used to achieve MALDI MS imaging measurements. The mass spectrometer was operated in positive-ion mode at a mass resolution of 140000 at $\mathrm{m} / z=200$. A nitrogen 
laser $(\lambda=337 \mathrm{~nm})$, operating at a repetition rate of $60 \mathrm{~Hz}$, was used for desorption/ionization. Mass accuracy better than $2 \mathrm{ppm}$ was achieved, and DHB/CHCA mixture was used as a matrix.

\section{6 cLIFT machine setup ${ }^{[10]}$}

An acousto-optic modulator (1002AF1, Polytec GmbH, Germany) to switch the laser (FSDL532-1000T, 1 W, Frankfurt Laser Company), a laser scanning system (hurrySCAN 10, Scanlab AG, Germany), an x-y microscope stage (SCANplus 100 x 100, Maerzhaeuser, Germany) and a camera (DCC1645C, Thorlabs Inc., Newton, NJ, USA) with a microscope lens (PLN 4XCY, Olympus GmbH, Hamburg,Germany) were used. cLIFT technique parameters: laser power $100 \%=320 \mathrm{~mW}$, irradiation time: $0.1-20 \mathrm{~ms}$.

\section{Fluorescence scanner}

Analysis of fluorescently labeled synthesis surfaces was performed with an InnoScan 1100 AL (Innopsys, France), with the wavelengths $532 \mathrm{~nm}$ and $635 \mathrm{~nm}$. ImageJ or IrfanView was used to enhance contrast and brightness of the scan images.

\section{Author Contributions}

D.S.M. developed the peptoid array synthesis with cLIFT and implemented the synthesis of bromoacetic-2,4-dinitrophenolester and the peptoids. B.St. implemented the synthesis of the peptoids. D.S.M. performed the analysis of the data, the MALDI measurements of the crude product and wrote the manuscript. B.St. and S.W.M. synthesized two Fmoc- $N$-substituted glycines. J.G. supported the synthesis of the peptoids. C.v.K. made the markers on the surfaces. B.Sp. set up and supported the MALDI imaging studies. D.R.B. performed the MALDI imaging and the analysis. T.F. built up the cLIFT setup and supported the 
implementation. B.R. supported the implementation and corrected the manuscript. F.F.L. supported the project, developed the cLIFT method and corrected the manuscript. F.B., A.N.M., S.B., U.S., B.R. supported the project and corrected the manuscript. B.Sp., S.W.M., D.R.B. corrected the manuscript.

\section{References}

[1] J. Striffler, D. S. Mattes, S. Schillo, B. Munster, A. Palermo, B. Ridder, A. Welle, V. Trouillet, V. Stadler, G. Markovic, G. Proll, S. Brase, F. F. Loeffler, A. NesterovMuller, F. Breitling, Chemnanomat 2016, 2, 897-903.

[2] F. F. Loeffler, T. C. Foertsch, R. Popov, D. S. Mattes, M. Schlageter, M. Sedlmayr, B. Ridder, F. X. Dang, C. von Bojnicic-Kninski, L. K. Weber, A. Fischer, J. Greifenstein, V. Bykovskaya, I. Buliev, F. R. Bischoff, L. Hahn, M. A. Meier, S. Brase, A. K. Powell, T. S. Balaban, F. Breitling, A. Nesterov-Mueller, Nat. Commun. 2016, 7 , 11844.

[3] J. E. Hein, V. V. Fokin, Chem.Soc. rev. 2010, 39, 1302-1315.

[4] a) N. Heine, T. Ast, J. Schneider-Mergener, U. Reineke, L. Germeroth, H. Wenschuh, Tetrahedron 2003, 59, 9919-9930 b) H. Ammann, G. Dupuis, Can. J. Chem. 1988, 68, 1651-1655;. c) P. Cuatrecasas, M. Wilchek, C. B. Anfinsen, Proc. Natl. Acad. Sci. U. S. A. 1968, 61, 636-643; d) H. N. Heine, PhD thesis, Humboldt-Universität (Berlin), 2000.

[5] J. A. W. Kruijtzer, L. J. F. Hofmeyer, W. Heerma, C. Versluis, R. M. J. Liskamp, Chem-Eur J 1998, 4, 1570-1580. 
